\title{
The Mus musculus papillomavirus type 1 E7 protein binds to the retinoblastoma tumor suppressor - implications for viral pathogenesis
}

Tao Wei ${ }^{\mathrm{a}}$, Miranda Grace ${ }^{\mathrm{b} \$}$, Aayushi Uberoi ${ }^{\mathrm{a} *}$, James C. Romero-Masters ${ }^{\mathrm{a}}$, Denis Lee ${ }^{\mathrm{a}}$, Paul F. Lambert $^{\text {a\# }}$, Karl Munger ${ }^{\text {b\# }}$

${ }^{a}$ McArdle Laboratory for Cancer Research, University of Wisconsin School of Medicine and Public Health, Madison, WI

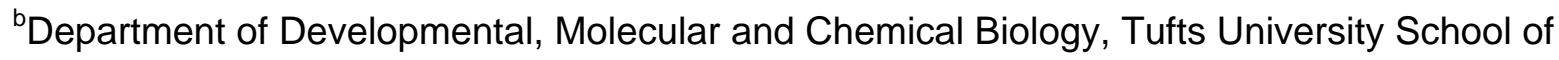
Medicine, Boston, MA

\$these two authors have contributed equally

${ }^{*}$ Current address: Department of Dermatology, University of Pennsylvania, Philadelphia, PA

\#Corresponding authors: Karl.Munger@tufts.edu

Plambert@wisc.edu

Running title: MmuPV1 E7 and viral pathogenesis

Keywords: Papillomavirus; viral pathogenesis, retinoblastoma tumor suppressor, E7, MmuPV1 


\section{Abstract}

2 The species specificity of papillomaviruses has been a significant roadblock for performing in

3 vivo pathogenesis studies in common model organisms. The Mus musculus papillomavirus type

41 (MmuPV1) causes cutaneous papillomas that can progress to squamous cell carcinomas in

5 laboratory mice. The papillomavirus E6 and E7 genes encode proteins that establish and

6 maintain a cellular milieu that allows for viral genome synthesis and viral progeny synthesis in

7 growth-arrested, terminally differentiated keratinocytes. The E6 and E7 proteins provide this

8 activity by binding to and functionally reprogramming key cellular regulatory proteins. The

9 MmuPV1 E7 protein lacks the canonical LXCXE motif that mediates the binding of multiple viral oncoproteins to the cellular retinoblastoma tumor suppressor protein, RB1. Our proteomic experiments, however, revealed that MmuPV1 E7 still interacts specifically with RB1. We show that MmuPV1 E7 interacts through its C-terminus with the C-terminal domain of RB1. Binding of MmuPV1 E7 to RB1 did not cause significant activation of E2F-regulated cellular genes. MmuPV1 E7 expression was shown to be essential for papilloma formation. Experimental infection of mice with MmuPV1 virus expressing an E7 mutant that is defective for binding to RB1 caused delayed onset, lower incidence, and smaller sizes of papillomas. Our results demonstrate that the MmuPV1 E7 gene is essential and that targeting non-canonical activities of RB1, which are independent of RB1's ability to modulate the expression of E2F-regulated genes, contribute to papillomavirus-mediated pathogenesis.

Importance

Papillomavirus infections cause a variety of epithelial hyperplastic lesions, warts. While most warts are benign, some papillomaviruses cause lesions that can progress to squamous cell carcinomas and approximately $5 \%$ of all human cancers are caused by human papillomavirus

24 (HPV) infections. The papillomavirus E6 and E7 proteins are thought to function to reprogram 25 host epithelial cells to enable viral genome replication in terminally differentiated, normally 
26 growth-arrested cells. E6 and E7 lack enzymatic activities and function by interacting and

27 functionally altering host cell regulatory proteins. Many cellular proteins that can interact with E6

28 and E7 have been identified, but the biological relevance of these interactions for viral

29 pathogenesis has not been determined. This is because papillomaviruses are species-specific

30 and do not infect heterologous hosts. Here we use a recently established mouse papillomavirus

31 (MmuPV1) model to investigate the role of the E7 protein in viral pathogenesis. We show that

32 MmuPV1 E7 is necessary for papilloma formation. The retinoblastoma tumor suppressor protein

33 (RB1) is targeted by many papillomaviral E7 proteins, including cancer-associated HPVs. We

34 show that MmuPV1 E7 can bind RB1 and that infection with a mutant MmuPV1 virus that

35 expresses an RB1 binding defective E7 mutant caused smaller and fewer papillomas that arise

36 with delayed kinetics. 


\section{INTRODUCTION}

Papillomaviruses (PVs) have been isolated from a wide range of vertebrate species. They have a tropism for squamous epithelia, and individual genotypes often have a marked preference for infecting mucosal or cutaneous squamous epithelia. Approximately 440 human PVs (HPVs) have been identified and they are phylogenetically classified into several genera (1). Among these, most alpha genus HPVs preferentially infect mucosal epithelia. A group of approximately 15 "high-risk" alpha genus HPVs are the etiological agents of almost all cervical cancers and a large percentage of other anogenital tract carcinomas as well as a growing fraction of head and neck squamous cell carcinomas (SCCs), particularly oropharyngeal cancers $(2,3)$. Overall, high-risk HPV infections contribute to $>5 \%$ of all human cancers (4). The beta and gamma genus HPVs mostly infect cutaneous epithelia and infections with some of these HPVs contribute to the development of cutaneous squamous cell carcinomas (cSCCs) in individuals afflicted by the rare hereditary disease, epidermodysplasia verruciformis $(5,6)$, or in long-term systemically immune-suppressed organ transplant patients (7-9).

Cell- and animal model-based studies have revealed that the E6 and E7 proteins of high-risk alpha, as well as the cSCC-associated beta HPVs, have oncogenic activities. HPV E6 and E7 encode small, cysteine-rich, metal-binding proteins. They lack intrinsic enzymatic activities, do not function as DNA binding transcription factors, and do not share extensive sequence

57 similarities with cellular proteins. By binding to and interfering with the functionality of important,

58 host regulatory proteins they elicit profound alterations in cellular physiology to permit long-term

59 viral persistence as well as viral progeny synthesis $(10,11)$. In rare cases, these alterations and

60 the cellular responses that are triggered can cause cancer formation (12). A large number of

61 potential cellular protein targets have been identified for the high-risk alpha HPV E6 and E7

62 proteins through proteomic studies $(10,11)$. Similar proteomic experiments with the beta HPV 
E6 and E7 proteins have revealed that, while they share some interactors with high-risk

mucosal HPVs, they also interact with distinct cellular proteins and signaling pathways (13).

Papillomaviruses are species-specific and cannot productively infect heterologous host organisms. Hence the biological relevance of specific interactions of the HPV E6 and E7 proteins with specific host pathways cannot be tested in infectious animal models. Traditional animal models of PV infection and pathogenesis are limited to species that are not genetically tractable. The discovery of the Mus musculus papillomavirus type 1 (MmuPV1), which can be used to experimentally infect standard laboratory mice, has finally provided a viable experimental model system to explore the importance of specific virus-host interactions in viral pathogenesis. MmuPV1 was discovered based on its ability to cause cutaneous papillomas and provides us an opportunity to better understand molecular mechanisms by which HPVs promote cutaneous disease in an in vivo animal model (14).

We have previously reported that the MmuPV1 E6 protein shares with the beta HPV8 E6 protein the ability to inhibit NOTCH and TGF-beta signaling by interacting with the NOTCH co-activator MAML and the DNA binding SMAD2 and SMAD3 proteins that are downstream of TGF-beta signaling (15). Moreover, by experimentally infecting with MmuPV1 mutant genomes we showed that the presence of a functional MAML1 binding site on E6 is critical for papilloma

82 formation (15). We have now extended these proteomic studies to the MmuPV1 E7 protein.

83 Here we show that MmuPV1 E7 expression is necessary for papilloma formation. Like some 84 gamma HPV E7 proteins, MmuPV1 E7 lacks an LXCXE (L, leucine; C, cysteine; E, glutamic acid; X, any amino acid)-based binding site for the retinoblastoma tumor suppressor, RB1 (16, 17). Using affinity purification of MmuPV1 E7 associated cellular protein complexes followed by

87 mass spectrometry, we discovered that MmuPV1 E7 can bind RB1. Similar to some animal PV 88 and gamma HPV E7 proteins that also bind RB1 despite lacking LXCXE domains, the RB1 
binding site maps to the MmuPV1 E7 C-terminus. Experimental MmuPV1 infection of a mouse strain that expresses an LXCXE protein binding deficient RB1 mutant, causes the formation of papillomas. This result shows that the ability of MmuPV1 to cause papillomas is not dependent on binding RB1 through its LXCXE binding cleft. Consistent with this finding, we mapped the MmuPV1 E7 binding site to the RB1 C-terminus. Unlike LXCXE containing E7 proteins, MmuPV1 E7 expression did not trigger efficient activation of E2F-responsive cellular genes. Experimental infection with a MmuPV1 mutant virus that encodes an RB1 binding defective E7 protein, inefficiently caused papillomas when compared to the wild type virus, and the lesions that did arise were smaller and appeared later than those arising in wild type MmuPV1 infected animals. These findings support the hypothesis that MmuPV1 E7 contributes to pathogenesis, at least in part, through its interactions with RB1.

\section{RESULTS}

\section{MmuPV1 E7 is required for viral pathogenesis}

To understand how MmuPV1 mediates its pathogenesis, we first asked whether the viral E7 gene is required for the virus to induce papillomas. To address this question, we engineered a stop codon immediately after the ATG initiation codon in the MmuPV1 E7 translational open reading frame in the context of the full-length MmuPV1 DNA genome. MmuPV1 E7 only contains one translational start codon, so no E7-related polypeptides can be expressed from internal methionine residues. Nor have there been identified any spliced MmuPV1 mRNAs that could produce E7-related polypeptide initiating from a start codon present in an upstream open reading frame (18). MmuPV1 quasivirus containing the wild type or E7 stop mutant were

111 generated in vitro in 293FT cells as described previously (19). The yield of virus particles

112 containing encapsidated viral genomes was determined by quantifying the amount of DNAse

113 resistant viral genomes in the fractions from the density gradient (19). These virus particles are

114 referred to as "quasiviruses" to distinguish them from authentic viruses generated in naturally 
infected tissue. The infectivity of these viral stocks was confirmed by RT-PCR detection of viral E1^E4 spliced mRNAs expressed at 48 hours after infecting cultured mouse keratinocytes

(Figure 1A).

Stocks of quasiviruses containing either wild type (MmuPV1) or E7-null (MmuPV1 E7 $7^{\text {STOP }}$ ) genomes were used to infect Nude-FoxN1 ${ }^{\text {nu/nu }}$ mice at cutaneous sites, both on the ears and tail, at the same dose of $10^{8}$ viral genome equivalents (VGE) that was shown previously to induce papillomas at $100 \%$ of sites infected with wild type MmuPV1 (20). Briefly, sites were wounded by lightly scarifying the epidermis with a needle, then a solution containing quasivirus was applied to the wounded skin. Mice were monitored for papilloma formation weekly for 3 months. As observed before (20), wild-type MmuPV1 quasivirus caused papilloma formation at $100 \%$ of infected sites on the nude mice, whereas infections with the mutant MmuPV1 E7 ${ }^{\text {STOP }}$ quasivirus did not induce any papillomas (Figure 1B). Mock-infected nude mice also did not develop papillomas (15). The experiment was repeated by placing on wounded sites naked recircularized viral genomes, which have been reported to be infectious and cause papilloma formation $(13,21)$. Consistent with our previous findings (15), $10 \mu \mathrm{g}$ of re-circularized wild type MmuPV1 genome caused papillomas at $100 \%$ of sites exposed to the viral DNA on the nude mice by the end of 3 months post-infection, whereas $10 \mu \mathrm{g}$ of re-circularized MmuPV1 E7 ${ }^{\text {STOP }}$ viral DNA failed to induce papillomas at any sites (Figure 1B). Together, these results indicate that the expression of the viral E7 protein is required for MmuPV1 to induce papillomatosis in vivo.

\section{MmuPV1 E7 lacks an LXCXE motif but can bind to RB1}

The retinoblastoma tumor suppressor, RB1, is an important cellular target of many PV E7 proteins. Most PV E7 proteins interact with RB1 through a conserved, N-terminal LXCXE motif (11). The MmuPV1 E7 protein, however, lacks an LXCXE sequence (Figure 2A). Some PVs 
141 including the canine papillomavirus type 2 (CPV2) and the gamma HPV4 and HPV197 E7

142 proteins have been shown to bind RB1 despite lacking LXCXE domains $(16,17)$. To determine

143 whether MmuPV1 E7 may bind RB1 we performed affinity purification/mass spectrometry

144 (AP/MS) experiments. N-terminal and C-terminal FLAG/HA epitope-tagged MmuPV1 E7

145 proteins were transiently expressed in HCT116 human colon carcinoma cells. HCT116 cells are

146 used because they are of epithelial origin, are highly transfectable, express wild-type RB1 and

147 do not contain any known exogenous viral sequences. MmuPV1 E7-associated proteins

148 complexes were isolated by affinity chromatography on HA antibody resin, eluted with HA

149 peptide, and analyzed by mass spectrometry. These experiments revealed that MmuPV1 E7

150 can interact with RB1 as evidenced by the detection of 34 and 16 unique RB1 peptides in

151 experiments performed with $\mathrm{N}$-terminally and C-terminally tagged MmuPV1 $\mathrm{E}$, respectively

152 (Table S1). We confirmed the MmuPV1 E7/RB1 interaction by transfecting HCT116 cells with

an expression vector encoding N-terminally FLAG/HA-tagged MmuPV1 E7 followed by

immunoprecipitation/western blot analysis (Figure 2B). We also found MmuPV1 E7 to bind to

endogenous murine Rb1 in similar IP/western experiments performed in mouse NIH3T3 necessarily carried out using expression vectors for wild-type or mutant forms of human RB1. domain, has been reported not only to bind RB1 but also to destabilize it (16). Given the

164 importance of RB1 destabilization by high-risk HPVs in cellular transformation (22), we asked

165 whether MmuPV1 E7 can destabilize RB1. RB1 was co-transfected in combination with

166 increasing amounts of MmuPV1 E7 into SAOS-2 human osteosarcoma cells, which express an 
inactive, C-terminally truncated, barely detectable RB1 mutant (23). In contrast to HPV16 E7 (22, 24), expression of MmuPV1 E7 did not cause a significant decrease in RB1 steady-state levels. Hence MmuPV1 does not cause detectable RB1 destabilization (Figure 2D).

\section{MmuPV1 E7 does not as efficiently activate E2F-dependent gene expression as HPV16 E7}

One of the best-studied biological activities of RB1 is its regulation of the activity of the transcription factor E2F1 (25-27). RB1 undergoes cell cycle-dependent phosphorylation and dephosphorylation. Hypophosphorylated RB1 binds to E2F family members and the resulting RB1/E2F transcriptional repressor complexes restrain transition from the $\mathrm{G} 1$ to the $\mathrm{S}$ phase of the cell cycle. When RB1 is hyperphosphorylated by cyclin-dependent kinases, it permits E2Fs to function as transcriptional activators and drive S-phase progression $(28,29)$. Like adenovirus E1A and polyomavirus large tumor antigens, LXCXE motif-containing HPV E7 proteins bind $\mathrm{RB} 1$ and abrogate the formation of RB1/E2F repressor complexes thereby causing aberrant Sphase entry. It is thought that this activity of the viral proteins is key to retaining infected host cells in a replication-competent state that is conducive for viral genome synthesis (30). To determine whether MmuPV1 binding to RB1 affects E2F transcription factor activity, we determined expression levels of four well-established, E2F-regulated genes, cyclin E2 (CCNE2) (31), minichromosome maintenance complex components 2 (MCM2) and 7 (MCM7) (32) as well as Proliferating Cell Nuclear Antigen (PCNA) (33) in MmuPV1 E7 expressing, telomeraseimmortalized human keratinocytes (iHFKs) or early passage mouse keratinocytes. HPV16 E7 expressing cells were used as controls. While expression of CCNE2, MCM2, MCM7, and PCNA was significantly increased in HPV16 E7-expressing iHFKs, there was no comparable increase in the expression of these genes in MmuPV1 E7-expressing iHFKs (Figure 3A) or primary mouse keratinocytes (Figure 3B). Based on these results, we conclude that MmuPV1 E7 does not as efficiently activate the expression of E2F-responsive genes as HPV16 E7. 
The MmuPV1 E7 protein interacts with RB1 sequences that are distinct from the LXCXE binding cleft

Because MmuPV1 E7 does not contain an LXCXE sequence, we wanted to test whether it binds RB1 similarly or distinctly to LXCXE-containing E7 proteins. The LXCXE binding site in RB1 has been determined by X-ray co-crystallography studies (34). Based on this information, an LXCXE binding defective RB1 mutant with amino acid substitutions at three critical contact residues (I753A; N757A; M761A) (RB1 ${ }^{\mathrm{L}}$ ) was constructed (35). We compared the abilities of HPV16 E7, which binds RB1 through its LXCXE motif, and MmuPV1 E7 to bind to wild-type $\mathrm{RB} 1$ versus the $\mathrm{RB} 1^{\mathrm{L}}$ mutant by expressing the corresponding proteins in SAOS-2 cells. As expected (35), HPV16 E7 interacted with wild-type RB1 but not the RB1 ${ }^{\mathrm{L}}$ mutant. In contrast, MmuPV1 interacted with both wild-type RB1 and mutant $R B 1^{L}$ with similar efficiencies. These experiments reveal that MmuPV1 interacts with RB1 sequences that are distinct from those necessary for interaction with LXCXE motif-containing E7 proteins (Figure 4).

\section{MmuPV1 causes warts in mice expressing the LXCXE binding defective $\mathrm{Rb}^{\mathrm{L}}$ mutant} MmuPV1 E7 can interact with the RB1 ${ }^{\mathrm{L}}$, we asked if MmuPV1 can cause disease in $R b 1^{L}$ mice. Ears of both wild-type $F V B$ and $R b 1^{L} F V B$ mice were infected with $10^{8}$ VGE of MmuPV1 (virus stock generated from MmuPV1-induced warts from a nude mouse - see Materials and Methods) as described previously (20) using the same methodology used in our previous experiments for quasivirus infections. By the end of 4 months, MmuPV1 caused a similar incidence of papillomas in both the wild type and $R b 1^{L} F V B$ mice (Figure 5A, $p$-value $=1$, two-sided Fisher's exact test). There was no apparent size difference between warts from the MmuPV1-infected

217 wild-type mice and those from $R b 1^{L}$ mice, based on H\&E analysis (data not shown). 
We next performed immunohistochemistry on tissues obtained at the experimental endpoint to compare the expression of biomarkers for viral infection between the papillomas arising in wild

221 type and $R b 1^{L}$ FVB mice (Figure 5B). Ki67-specific immunohistochemistry showed similar

222 levels of cell proliferation in the papillomas arising on both the wild type and $R b 1^{L} \mathrm{FVB}$ mice.

223 MCM7 was also upregulated to similar levels, indicating that MmuPV1 is capable of increasing

224 E2F-driven gene expression despite the disruption in the LXCXE binding cleft in the $R b 1^{L} F V B$

225 mice. These results demonstrate that the disruption of the ability of RB1 to bind proteins via

226 their LXCXE-motifs is not required for MmuPV1 to induce papillomas or to induce expression of

227 E2F-responsive genes.

\section{C-terminal RB1 sequences are necessary for interaction with MmuPV1 E7}

Given that MmuPV1 E7 does not interact with the LXCXE binding cleft of RB1 we mapped the RB1 region responsible for binding MmuPV1 E7. We co-expressed MmuPV1 E7 with plasmids expressing full-length RB1 (amino acid residues 1-928) or truncation mutants of RB1 lacking the amino-terminus (amino acid residues 379-928) or the C-terminus (amino acid residues 1-792) in SAOS2 human osteosarcoma cells. HPV16 E7 was used as a control. As expected, HPV16 E7 efficiently bound wild-type RB1, as well as the two truncation mutants, both of which encode the efficiently interact with the 1-792 mutant RB1 that lacks the C-terminal domain of RB1 (Figure 6A, B). Hence MmuPV1 E7 primarily interacts with the C-terminal domain of RB1.

\section{C-terminal MmuPV1 E7 sequences are necessary for RB1 binding}

241 It has previously been reported that the E7 proteins of CPV2 and HPV4, which also lack LXCXE

242 motifs in their N-termini, associate with RB1 through their C-termini (16). Hence, in addition to

243 testing some mutations in the N-terminus of MmuPV1 E7, we also generated MmuPV1 E7

244 mutants in the C-terminal domain. We focused on regions that are conserved between CPV2, 
HPV4, HPV197, and MmuPV1 (Figure 7A). Of all the mutants that were tested, a four amino acid deletion of residues 84 to 87 (MmuPV1 E7 $7^{\Delta 84-87}$ ) and an alanine substitution at aspartate residue 90 (MmuPV1 E7 ${ }^{\text {D90A }}$ ) were found to be defective for RB1 binding (Figure 7B). Based on these results we generated two additional substitution mutants, MmuPV1 E7 ${ }^{\text {D90T }}$ and MmuPV1 $E 7^{\mathrm{D} 90 \mathrm{~N}}$. MmuPV1 $\mathrm{E} 7^{\mathrm{D90T}}$ was generated to mimic the HPV16 E7 threonine residue at this position (Figure 7A). MmuPV1 E7 ${ }^{\mathrm{D} 90 \mathrm{~T}}$ displayed decreased RB1 binding similar to MmuPV1 $\mathrm{E} 7^{\mathrm{D} 90 \mathrm{~A}}$. The MmuPV1 $\mathrm{E} 7^{\mathrm{D} 90 \mathrm{~N}}$ mutant, which was generated to neutralize the negative charge while maintaining the general architecture of the side chain, retained some binding to RB1, even MmuPV1 E7 ${ }^{\mathrm{D} 90 \mathrm{~A}}$ mutant was also defective for binding to murine Rb1 (Figure 7D). Hence, similar to CPV2 and gamma-HPVs (16), MmuPV1 E7 binds to RB1 through its C-terminal domain, and based on the results described above, we chose the MmuPV1 E7 ${ }^{\mathrm{D} 90 \mathrm{~A}}$ mutant for our follow-up studies.

\section{Reduced incidence and smaller sized warts in MmuPV1 E7 $7^{\mathrm{D90A}}$ infected mice}

To assess whether E7's ability to bind Rb1 contributes to MmuPV1 pathogenesis, we introduced the D90A mutation into the complete MmuPV1 DNA genome which we used to make quasivirus particles in 293FT cells. We then characterized the ability of this mutant MmuPV1 versus wild-

263 type MmuPV1 to cause papillomatosis in mice. Quasivirus stocks were confirmed to be

264 infectious (Figure 8A), by exposing mouse keratinocytes to the quasiviruses and 48 hours later 265 harvesting RNA to detect the presence of viral E1^E4 spliced transcripts by RT-PCR. We then 266 performed in vivo infections with these stocks of infectious quasivirus. 6-8 weeks old Nude-

267 FoxN1 $1^{n u / n u}$ mice were scarified on their ears and tails and infected with wildtype MmuPV1 or $\mathrm{E} 7^{\mathrm{D} 90 \mathrm{~A}}$ mutant MmuPV1 quasivirus at doses of either $10^{7}$ (stock 2 ) or $10^{8}$ VGE (stock 1 ).

269 Papilloma incidence was monitored biweekly for 4 months. At the endpoint, wild-type MmuPV1 
271 dose of MmuPV1 E7 ${ }^{\mathrm{D} 90 \mathrm{~A}}$ quasivirus caused papillomas at a significantly lower frequency, $12 \%$

272 (Figure 8B, p<0.0001, two-sided Fisher's exact test). At the lower $10^{7}$ VGE dose, wild-type

273 MmuPV1 caused papillomas at a 65\% frequency, whereas MmuPV1 E7 ${ }^{\mathrm{D} 90 \mathrm{~A}}$ caused papillomas

274 in only $8 \%$ of infected sites (Figure 8B, p<0.001, two-sided Fisher's exact test). At both doses,

275 the papillomas arising on mice infecting with MmuPV1 E7 ${ }^{\mathrm{D} 90 \mathrm{~A}}$ appeared at later time points

276 (Figure 8C, MmuPV1 (10 ${ }^{8}$ VGE) vs. MmuPV1 E7 ${ }^{\text {D90A }}$ stock 1, p<0.0001; MmuPV1 (10 ${ }^{7}$ VGE)

277 versus MmuPV1 E7 ${ }^{\mathrm{D} 90 \mathrm{~A}}$ stock 2, p<0.0001 two-sided LogRank test). At the 4 month endpoint,

278 we harvested the papillomas from all infected mice, fixed and serially sectioned the lesion, and

279 performed H\&E staining. We performed scans of the H\&E-stained sections from 6 representative papillomas induced by MmuPV1 ( 3 from $10^{8}$ VGE and 3 from $10^{7}$ VGE), and 3 papillomas induced by MmuPV1 E7 ${ }^{\mathrm{D} 90 \mathrm{~A}}$ quasiviruses (we scanned all three papillomas that arose on mice infected with the MmuPV1 E7 ${ }^{\mathrm{D} 90 \mathrm{~A}}$ quasivirus, regardless of virus dose) (Figure

8D). The size of each papilloma was assessed using ImageScope under the same quasiviruses and confirmed that warts indeed contained the expected virus and that no crosscontamination had occurred. Together, the assessments of the incidence of papillomas, the time of onset of papillomas, and the size of papillomas at the endpoint all indicate that the interaction

292 between MmuPV1 E7 and Rb1 quantitatively correlates with the MmuPV1's ability to cause 293 papillomatosis. 
297 To determine whether the papillomas caused by MmuPV1 E7 ${ }^{\mathrm{D} 90 \mathrm{~A}}$ displayed similar or different

298 microscopic features compared to those caused by wild-type MmuPV1, we performed

299 immunohistochemistry to assess the expression of biomarkers for papillomavirus-associated

300 lesions. Evidence for productive viral infections within the papillomas was scored by performing

301 immunofluorescence staining to detect the viral capsid protein L1 (Figure 9, panel B). L1

302 expression was similar in papillomas induced by the wild type and mutant quasiviruses.

303 Papillomas induced by wild type and E7 $7^{\mathrm{D} 90 \mathrm{~A}}$ quasiviruses also showed similar patterns of

304 keratinocyte differentiation with cytokeratin 14 upregulated in the suprabasal layers of the

305 papillomas (Figure 9, panel B), indicating similar delays in terminal differentiation. MCM7, an

306 E2F-responsive gene that is upregulated in papillomavirus-related lesions caused by high-risk

307 HPV (38) and MmuPV1 (39) infections, was similarly upregulated in papillomas induced by wild

308 type and $E 7^{\mathrm{D} 90 \mathrm{~A}}$ quasiviruses, indicating increased levels of E2F-mediated transcription in both

309 cases (Figure 9, panel C). The incorporation of BrdU into genomic DNA is often upregulated in

310 papillomavirus-related lesions as a consequence of increased DNA synthesis. Both MmuPV1

311 and MmuPV1 E7 $7^{\mathrm{D} 90 \mathrm{~A}}$ - induced papillomas showed increased levels of BrdU, with no obvious

312 differences in abundance or localization of BrdU-positive cells within the papillomas, indicating

313 similarly enhanced levels of DNA synthesis (Figure 9, panel D). Based on the biomarkers

314 tested, there were no significant differences in the histopathological features due to the loss of

315 interaction between E7 and Rb1.

\section{DISCUSSION}

318 The species specificity of papillomaviruses has greatly limited studies of how specific

319 biochemical activities of individual viral proteins contribute to viral pathogenesis in a natural

320 infection model. The discovery of MmuPV1 and its ability to infect and cause papillomas in

321 laboratory mice has removed this barrier. MmuPV1 is a member of the Pi genus, which 
encompasses rodent PVs (1) and is most related to the cutaneous beta- and gamma-genus HPVs (40).

The MmuPV1 E6 and E7 proteins share sequence similarities to cutaneous beta- and gammaHPVs, respectively (41). We have previously reported that MmuPV1 E6 is necessary for papilloma formation in experimentally infected mice (15). MmuPV1 E6 shares key cellular targets and biological activities with the beta-HPVs 5 and 8 E6 proteins that affect key tumor suppressor gene functions, including the ability to bind the NOTCH transcriptional coactivator MAML1 and the SMAD2 and SMAD3 mediators of transforming growth factor-beta (TGF-beta) signaling (15). In particular, we have shown that MmuPV1 E6's ability to bind NOTCH correlates with MmuPV1's ability to cause disease (15).

Here we show that similar to what we previously reported for E6 (15), MmuPV1 E7 is also necessary for papilloma formation. Like CPV2 E7 and some gamma-HPV E7 proteins, the MmuPV1 E7 protein lacks an N-terminal LXCXE motif, which is present in multiple viral and cellular proteins where it serves as the binding site for members of the retinoblastoma tumor suppressor family (40). We provide evidence that MmuPV1 E7 can bind to both the human and murine retinoblastoma tumor suppressor proteins. We determined that the RB1 binding site is located in the MmuPV1 E7 C-terminal domain, similar to CPV2 and gamma-HPV4 E7 proteins (16). We identified a C-terminal mutant, MmuPV E7 ${ }^{\mathrm{D} 90 \mathrm{~A}}$, that was markedly reduced for RB1 binding. The HPV16 E7 C-terminus may also contain a low-affinity RB1 binding site (42) and mutation of the T86 residue in HPV16 E7 (which corresponds to MmuPV1 E7 D90) to an aspartate (as present in MmyPV1) did not significantly affect RB1 binding in a yeast two-hybrid format (43), whereas the MmuPV1 E7 E7 ${ }^{\mathrm{D} 90 \mathrm{~T}}$ mutant exhibited decreased RB1 binding. Infection with the MmuPV1 E7 $7^{\mathrm{D} 90 \mathrm{~A}}$ mutant revealed that RB1 binding correlates with MmuPV1's ability to promote efficient papilloma formation in the cutaneous infection model. However, it does not appear to be essential for pathogenesis as small warts did arise, albeit at significantly reduced 
efficiency and with a later time of onset. There is precedence for these findings as studies that were done in cottontail rabbit PV (SfPV1, a.k.a. CRPV1) found E7 to be essential for promoting disease $(44,45)$ but that E7's ability to interact with RB1, albeit through an LXCXE sequence (46), is not essential for inducing papillomas in experimentally infected rabbits (47). In addition, studies with transgenic mice have also provided evidence that HPV16 E7 can cause hyperproliferation in mice that express the $R b 1^{L}$ mutant, which HPV16 E7 is unable to bind (36, 37). Therefore, it is likely that other biological activities of PV E7 are also playing important roles in promoting disease across various PVs.

PV E7 proteins with an LXCXE motif bind to a shallow cleft within the RB1 B "pocket" domain (34). In contrast, MmuPV1 E7 interacts with the RB1 C-terminal domain. Consistent with these results, experimental MmuPV1 infection of mice engineered to express the $R b 1^{L}$ allele, in which the LXCXE binding cleft in the B domain is mutated, caused papilloma formation at a similar efficiency as mice infected with wild type MmuPV1 genomes. The RB1 C-terminal domain is highly conserved amongst RB1 proteins from different species and is necessary for RB1 to induce permanent G1 growth arrest and senescence (48-50). It has been shown to mediate interactions with several different cellular proteins, including the ABL1 non-receptor tyrosine kinase (51), the F-box protein SKP2 (52) and a non-canonical E2F1 complex that contains the lysine methyltransferase $\mathrm{EZH} 2(53,54)$. It has been reported that $A B L 1$ selectively binds to hypophosphorylated RB1 and that RB1 binding inhibits $A B L 1$ enzymatic activity (51). While the RB1 C-terminus is necessary, the ABL1 binding sequences have not been mapped in detail and 367 the biological relevance of the RB1/ABL1 interaction has remained enigmatic. SKP2 is an F-box 368 protein that is part of the cullin1 based ubiquitin ligase complex that has been shown to control 369 the degradation of the CDK2 inhibitor, p27 $7^{\mathrm{KIP} 1}$ (CDKN1B). SKP2 is rapidly degraded during G1, when RB1 is hyperphosphorylated, by $\mathrm{CDH} 1$ containing anaphase-promoting complex or cyclosome (APC/C $\left.\mathrm{C}^{\mathrm{CD} 1}\right)$ (55) that binds to RB1's A-B pocket. However, when RB1 gets 
372 hyperphosphorylated, APC/C ${ }^{\mathrm{CDH} 1}$ dissociates from RB1 which leads to SKP2 degradation of

$373 \mathrm{p} 27^{\mathrm{KIP} 1}$ and increased CDK2 activity which promotes S-phase entry. These and other non-

374 canonical RB1 activities (56) including the ability of RB1 to interact with E2F1/EZH2 complexes

375 that are not involved in cell cycle regulation are likely targeted by MmuPV1 E7, but further

376 studies are necessary to identify the specific target(s) and to delineate the molecular

377 consequences of C-terminal RB1 binding.

378 Given that phosphorylation-specific RB1 binding and release of E2F transcription factor

379 complexes involve RB1 sequences within the A-B domain $(57,58)$, and that MmuPV1 E7

380 interacts with RB1 C-terminus, it was not surprising that MmuPV1 E7 expression did not cause

381 efficient activation of E2F-responsive genes. Nevertheless, MmuPV1 can induce expression of

382 MCM7, a strongly E2F-responsive gene, in vivo in the context of papillomas it induces. We also

383 observed MCM7 induction in MmuPV-1-induced papillomas arising in mice expressing the $R b 1^{L}$

384 allele and in papillomas arising in animals infected with MmuPV1 genomes expressing the RB1

385 binding defective $E 7^{\mathrm{D} 90 \mathrm{~A}}$ mutant. This raises the interesting question: which MmuPV1 protein(s)

386 is responsible for the increased MCM7 expression. Experiments performed with HPVs in cell

387 and transgenic animal-based models have all suggested that this activity is provided by E7 and

388 is based on its ability to inactivate RB family members and it is possible that $E 7^{\mathrm{D} 90 \mathrm{~A}}$ mutant

389 retains low-level RB1 binding that may be sufficient to cause some expression of E2F-

390 responsive genes in vivo. However, our study is not to first to document E7 independent

391 induction of hyperproliferation. Experimental infection of rabbits with an SfPV1 mutant virus that

392 expressed an RB binding-deficient E7 mutant still caused the emergence of papillomas (47).

393 Our work is entirely consistent with this observation; moreover, our studies provide no evidence 394 that MmuPV1 E7 can efficiently activate the expression of E2F-responsive genes. It is possible 395 that MmuPV1 encodes another protein that can activate E2F-dependent promoters through 
because it contains an LXCXE motif $\left(\mathrm{L}_{67} \mathrm{ACKE}_{71}\right)$ in between its two $(\mathrm{CXXC})_{2}$ zinc-binding domains; however, our prior AP/MS experiments failed to provide any evidence that MmuPV1 E6 binds to any of the RB family members (13). Another possibility is that the expression of E2F-responsive genes in the papillomas reflects the ability of MmuPV1 E6 to impede

401 keratinocyte differentiation through its inhibition of NOTCH and TGF-beta signaling which may 402 help MmuPV1 infected cells maintain a proliferative state (15). Regardless, given that MmuPV1

403 E7 expression is necessary for papilloma formation, it will be important to determine the mechanism by which MmuPV1 E7 contributes to papilloma formation. Infections with an RB1 binding defective E7 mutant gives rise to smaller papillomas with lower efficiency and delayed kinetics compared to papillomas caused by wild-type MmuPV1 infection. It will be important to determine whether papillomas that express the RB1 binding defective E7 mutant progress to cancer at a similar frequency, or at all, compared to papillomas caused by wild-type MmuPV1.

In summary, our results show that loss of RB1 binding by MmuPV1 E7 correlates with a quantitative defect in papilloma induction. Hence MmuPV1 E7 binding to RB1's C-terminal

411 domain remains an important mechanism by which MmuPV1 promotes disease. The integrity of

412 the RB1 C-terminus is important for many activities of RB1, but whether or how any of these

413 contribute to Rb1's tumor suppressor activity is largely unknown. Given, the vast majority of 414 studies on RB1 have focused on its ability to control E2F transcription factor activity, which is 415 shared with other RB family members that are not frequently mutated in tumors, it is unlikely 416 that regulation of E2F transcription factor activity is the sole tumor-suppressive function of RB1.

417 It will be important to rigorously determine which specific function of RB1's C-terminus

418 MmuPV1E7 disrupts. Such studies promise to provide exciting new insights into the molecular basis of RB1's tumor suppressor activity. 


\section{MATERIALS AND METHODS}

\section{Cells}

423 U2OS human osteosarcoma cells were obtained from ATCC and grown in Dulbecco's Modified

424 Eagle Medium (DMEM; Invitrogen) supplemented with 10\% fetal bovine serum (FBS). SAOS-2

425 human osteosarcoma cells were obtained from ATCC and grown in McCoy's 5A medium

426 (Invitrogen) supplemented with 15\% FBS. HCT116 human colon carcinoma cells were obtained

427 from ATCC and grown in McCoy's 5A medium (Invitrogen) supplemented with 10\% FBS. NIH

428 3T3 murine fibroblasts were obtained from the ATCC and grown in DMEM (Invitrogen)

containing 5\% FBS. JB6 mouse keratinocytes (gift from Dr. Nancy $\mathrm{H}$. Colburn, NCl) were

maintained in DMEM containing 5\%FBS. 293FT cells (ATCC) were maintained in DMEM with

10\%FBS and $300 \mu \mathrm{g} / \mathrm{mL}$ neomycin (G418). Telomerase-immortalized human foreskin

keratinocytes Cl398 (iHFK) (60) were a kind gift from Aloysius Klingelhutz (U. of lowa.). iHFK

lines expressing various E7 proteins were established by transducing iHFKs with the

437 incubation in PBS containing $10 \%$ antibiotics for 2 minutes, skin pieces were incubated in $0.25 \%$

438 trypsin overnight at $4^{\circ} \mathrm{C}$. The epidermis was then separated from the dermis using sterile

439 forceps, minced with a single edge razor blade, and then stirred for 1 hour at $37^{\circ} \mathrm{C}$ in $\mathrm{F}$-media

440 (61) to generate a single-cell suspension. The cells were strained using $0.7 \mu \mathrm{m}$ membrane

441 (102095-534; VWR ), and cultured in F-media (62) containing $10 \mu \mathrm{M}$ Y-27632 Rho-kinase

442 inhibitor (63) in the presence of mitomycin C (M4287; Sigma) treated 3T3 J2 fibroblasts. Early

443 passage cells were infected with the recombinant lentiviral or retroviral vectors expressing

444 HPV16 E7 or MmuPV1 E7, respectively, in F-medium in the absence of Y-27632 and 3T3 J2

445 feeders and re-infected after 24 hours. At 72 hours after the first infection, cells were selected 
with the appropriate antibiotics. After selection, cells were maintained in F-media containing 10 $\mu \mathrm{M}$ Y-27632 and mitomycin C-treated 3T3 J2 fibroblasts.

\section{Plasmids and antibodies}

450 The MmuPV1 E7 ORF was PCR amplified from the MmuPV1 genome and cloned into N- or C-

451 terminal FLAG/HA-CMV (64) and untagged pCMV-BamNeo (65) plasmids. Mutant MmuPV1 E7 452 constructs were generated by site-directed mutagenesis of N-FLAG/HA-mE7-CMV. pLenti-N-

453 mE7 was generated by Gateway cloning (Invitrogen) of PCR-amplified mE7 into pLenti 6.3/V5

454 DEST (Invitrogen). The RB1-truncation plasmids pSG5-HA-Rb 1-928, 1-792, and 379-928 were 455 kind gifts from Bill Sellers (Broad Institute). The pFADRb and pFADRbL plasmids were kindly provided by Fred Dick (Western University, Ontario). Other plasmids used were pLXSN HPV16

457 E7 (66), pCMV-Rb (67) (obtained from Phil Hinds, Tufts), CMV-C-16E7 (17), pBABE puro (68), and pEGFP-C1 (Clontech). The following primary antibodies were used for immunoprecipitations and western blotting: beta-Actin (MAB1501; Millipore), FLAG (F3165; Sigma), GFP (9996; Santa Cruz), HA (ab9110; Abcam), RB1 (Ab-5, OP66; Millipore), and Rb1

461 (SC-74570, Santa Cruz). Secondary anti-mouse or anti-rabbit antibodies conjugated to horseradish peroxidase were from GE Healthcare.

\section{Immunological Methods}

Affinity purification/mass spectrometry analyses of MmuPV E7 were performed as previously described (17). HCT116 cells were transfected using Polyethylenimine (PEI) (69). At 48 hr post-

467 transfection cells were harvested in EBC buffer (50mM Tris-Cl pH 8.0, $150 \mathrm{mM} \mathrm{NaCl}, 0.5 \%$ NP-

46840 and 0.5mM EDTA) supplemented with protease inhibitors (Pierce). Anti-Hemagglutinin (HA;

469 Sigma) or anti-Flag epitope (Sigma) antibodies coupled to agarose beads were used for

470 immunoprecipitations followed by SDS-PAGE and western blot analysis on PVDF membranes.

471 After incubation with appropriate primary and secondary antibodies, blots were visualized by 
472 enhanced chemiluminescence and images captured using a Syngene ChemiXX6 imager with

473 Genesys software version 1.5.5.0. Signals were quantified with Genetools software version

$474 \quad 4.03 .05 .0$.

475

RB1 Degradation assays

477 RB1 degradation assays were performed as previously described (22). SAOS-2 cells were

478 transfected with CMV-RB and varying amounts of pCMV N-FLAG/HA mE7. pCMV-C16 E7 was

479 used as positive control and pEGFP-C1 was co-transfected to assess transfection efficiency. At

48048 hours post-transfection cells were lysed in EBC and samples containing $100 \mu \mathrm{g}$ protein were

481 subjected to western blot analysis as described above.

482

\section{Quantitative Reverse Transcription PCR}

484 RNA was isolated from pLenti-N-FLAG/HA mE7, iHFK pLenti-C-FLAG/HA-16E7, and pLenti-NGFP infected iHFKs and pLenti-N-FLAG/HA mE7, pLXSP-16E7 or control vector infected

490 Target expression levels were normalized to GAPDH expression.

\section{Animals}

493 Immunodeficient Athymic Nude-FoxN1 ${ }^{n u / n u}$ mice were purchased from Envigo. $R B 1^{L}$ mutant

494 mice were maintained on the FVB background and genotyped as published previously $(37,70)$.

495 Mice were housed in the Association for Assessment of Laboratory Animal Care-approved

496 McArdle Laboratory Animal Care Unit. All procedures were carried out in accordance with an 
animal protocol approved by the University of Wisconsin Institutional Animal Care and Use Committee (IACUC; protocol number M005871).

\section{Infection of nude mice with MmuPV1 quasiviruses}

501 MmuPV1 quasiviruses (wild type, E7 ${ }^{\text {STOP }}, E 7^{\text {D90A; }}$ note that the term "quasivirus" is used in the 502 papillomavirus field to identify a virus that is generated in cells by co-transfection of viral genomes with a plasmid that expresses the viral capsid proteins) were generated as described before (15). Briefly, 293FT cells were transfected with a MmuPV1 capsid protein expression plasmid $(71,72)$ and the re-circularized genome, either from plasmids containing the wild type or mutant MmuPV1 genomes. After incubation at $37^{\circ} \mathrm{C}$ for 48 hours, cells were harvested and virus extracted. The amount of packaged viral DNA in the stocks of quasiviruses was quantified by Southern blotting allowing us to define the "viral genome equivalents" (VGE), as a measure of virus concentration in each stock. The quasiviruses were used to infect Nude-FoxN1 ${ }^{\text {nu/nu }}$ mice as previously described (15). Briefly, animals were placed under anesthesia and infected by first

511 scarifying the epidermis using a 27-gauge syringe needle and then pipetting onto the wounded

512 site the indicated amount of quasivirus using a siliconized pipette tip. Each mouse was infected 513 at 5 sites maximum (one site per ear, three sites on tail). Papillomatosis was monitored weekly/ 514 bi-weekly as indicated.

\section{Infection of FVB-background mice with MmuPV1}

517 The infection method has been described previously with some modifications (20). Briefly, under

518 anesthesia, mouse ears of both FVB and $R B 1^{L}$ mutant mice were scarified first using 27-gauge

519 syringe needles and infected with $10^{8} \mathrm{VGE} /$ site of a pre-prepared stock of MmuPV1 virus

520 generated from a MmuPV1-infected wart. 24 hours later, mice were exposed to 300mJ UVB

521 (Daavlin, Bryan, OH). Papillomatosis was monitored over 4 months. 


\section{RT-PCR to detect MmuPV1 E1^E4 spliced transcripts}

524 Mouse keratinocytes JB6 cells were infected with MmuPV1 wild type and mutant quasiviruses at

$52510^{8} \mathrm{VGE}$, and changed to fresh media 3 hours later. After incubation at $37^{\circ} \mathrm{C}$ for 48 hours, total

526 RNA was extracted from infected JB6 cells using the RNeasy kit (Qiagen) and reverse-

527 transcribed into cDNA using the QuantiTect Reverse Transcription Kit (Qiagen). E1^Е4

528 transcripts were detected by PCR, using p53 as a positive control. Primer sequences were

529 described previously (72).

\section{BrdU incorporation}

532 To evaluate levels of DNA synthesis, we performed bromodeoxyuridine (BrdU) incorporation by injecting BrdU (Sigma, dissolved in PBS to $12 \mathrm{mg} / \mathrm{ml}$ stock concentration, keep at $-20^{\circ} \mathrm{C}$ ). Mice were intraperitoneally injected with $250 \mu \mathrm{l}$ stock BrdU one hour before harvest. Tissues were harvest and processed for immunohistochemistry using a BrdU-specific antibody (203806, Calbiochem) as previously described (15).

\section{Histological analysis}

539 Tissues were harvested and fixed in 4\% paraformaldehyde (in PBS) for 24 hours, then switched

to $70 \%$ ethanol for 24 hours, processed, embedded in paraffin, and sectioned at $5 \mu \mathrm{m}$ intervals.

541 Every 10th section was stained with hematoxylin and eosin (H\&E).

\section{MmuPV1 L1-cytokeratin dual immunofluorescence and Immunohistochemistry}

544 L1 signals were detected using a tyramide-based signal amplification (TSA) method (73). A

545 detailed protocol is available at: https://www.protocols.io/view/untitled-protocol-i8cchsw.

546 For immunohistochemistry, tissue sections were deparaffinized in xylenes and rehydrated in

$547100 \%, 95 \%, 70 \%$, and $50 \%$ ethanol, then in water. Antigen unmasking was performed by

548 heating with $10 \mathrm{mM}$ citrate buffer $(\mathrm{pH}=6)$ for 20 minutes. Blocking was performed with $2.5 \%$ 
549 horse serum in PBST for 1 hour at room temperature (RT). Slides were incubated in primary

550 antibody (BrdU; MCM7, Thermo Scientific, Fremont, CA) at $4^{\circ} \mathrm{C}$, overnight in a humidified

551 chamber. M.O.M.® ImmPRESS® HRP (Peroxidase) polymer kit (Vector, MP-2400) was applied

552 the next day for 1 hour at RT for secondary antibody incubation. Slides were then incubated

553 with 3,3'-diaminobenzidine (Vector Laboratories), and counterstained with hematoxylin. All

554 images were taken with a Zeiss Axiolmager M2 microscope using AxioVision software version

$555 \quad 4.8 .2$.

556

557 Full scan for wart size measurement, and statistical analysis

558 Full scans of representative warts were performed by the UW Translational Research Initiatives

559 in Pathology (TRIP) facility. Measurements were performed on the full-scanned images using

560 ImageScope software (v12.4.0). All statistical analyses were performed using MSTAT statistical

561 software version 6.4.2 (http://www.mcardle.wisc.edu/mstat).

562

563

\section{ACKNOWLEDGMENTS}

564 We thank Dr. Al Klingelhutz (University of lowa) for providing telomerase immortalized human

565 foreskin keratinocytes, members of the Lambert and Munger labs for stimulating discussion and

566 valuable suggestions throughout the course of this work, Ella Ward-Shaw for expert

567 histotechnology assistance, and Simon Blaine-Sauer for isolating C57/B6 mouse keratinocytes

568 from neonates. Supported by PHS grant R01 CA228543 (K.M. and P.F.L.) and a Ruth L.

569 Kirschstein Postdoctoral Individual National Research Service Award F32 CA254019 (J.R.). 
571

572

573

574

575

576

577

578

579

580

581

582

583

584

585

586

587

588

589

590

591

592

593

594

595

596

\section{FIGURE LEGENDS:}

Figure 1. MmuPV1 lacking E7 expression does not induce warts. The MmuPV1 E7 ${ }^{\text {STOP }}$ quasivirus is infectious. To assess infectivity of quasivirus stocks, mouse JB6 keratinocytes were exposed to quasivirus and 48 hours later RNA was extracted and subjected to reverse transcription-coupled polymerase chain reaction to detect MmuPV1 E1^E4 transcripts (top panel). GAPDH expression is shown as a control (bottom panel). Samples were run on the same gel, with irrelevant lanes in the middle cropped out in (A). Tails and ears of nude mice were scarified and infected with the indicated amounts of quasiviruses or DNA and monitored for wart formation over 3 months. Neither MmuPV1 E7 ${ }^{\text {STOP }}$ quasivirus nor MmuPV1 E7 ${ }^{\text {STOP }}$ DNA induced wart formation, while wild type MmuPV1 quasivirus or DNA induced warts with a $100 \%$ penetrance $(\mathbf{B})$.

\section{Figure 2. MmuPV1 E7 binds but does not destabilize the retinoblastoma tumor} suppressor protein, RB1. Alignment of the N-terminal sequences of MmuPV1 E7 and the canine papillomavirus 2 (CPV2), $\gamma 1$-HPV4, $\gamma 24-H P V 197, \beta 1-H P V 8$, and $\alpha 9-H P V 16$. Identical residues are marked by black boxes, chemically similar residues are shaded in gray. The position of the LXCXE RB1 binding site is highlighted. A cartoon of the domain structure of E7 is shown on top, with the $\mathrm{N}$ - terminal sequences that show similarity the conserved regions 1 and 2 (CR1, CR2) of adenovirus E1A proteins indicated (A). MmuPV1 E7 (M E7) can interact with RB1 by immunoprecipitation/immunoblot analysis. Duplicate cultures of HCT116 cells were transfected with an N-terminally HA/FLAG epitope-tagged MmuPV1 E7 expression plasmid and co-precipitated RB1 protein detected by immunoblotting (B). MmuPV1 E7 (M E7) can interact with murine Rb1 by immunoprecipitation/immunoblot analysis. NIH 3T3 murine fibroblasts were transfected with an N-terminally HA/FLAG epitope-tagged MmuPV1 E7 expression plasmid and co-precipitated RB1 protein detected by immunoblotting (C). MmuPV1 E7 does not destabilize 
RB. SAOS-2 human osteosarcoma cells that do not express detectable endogenous RB were transfected with an RB expression vector and increasing amounts of an expression vector for $\mathrm{N}$ terminally HA/FLAG epitope-tagged MmuPV1 followed by western blotting to assess RB1 steady levels by western blotting. GFP was co-transfected and assessed by western blotting to

601 control for transfection efficiency. HPV16 E7 (16 E7) was used as a positive control. A

602 representative blot from one of four experiments is shown (D).

Figure 3. MmuPV1 E7 does not efficiently activate E2F-regulated genes. Expression of E2F target genes, cyclin E2 (CCNE2), Minichromosome Maintenance Complex Components 2 and 7 (MCM2, MCM7) and Proliferating Cell Nuclear Antigen (PCNA) in immortalized human foreskin keratinocytes (iHFKs) (A), or in primary mouse keratinocytes (MK) (B) transduced with control vector (C), MmuPV1 E7 (M E7) or HPV16 E7 (16 E7) as determined by quantitative reverse transcription-coupled polymerase chain reaction analysis ${ }^{* * \star *}, p<0.001 ;{ }^{* * *}, p<0.005$; n.s. not significant). wildtype RB1 or the $\mathrm{RB} 1^{\mathrm{L}}$ mutant that contains three amino acid mutations in the $\mathrm{LXCXE}$

615 binding cleft in combination with MmuPV1 or HPV16 E7 expression vectors. MmuPV1 E7 binds 616 wild type and $\mathrm{RB} 1^{\mathrm{L}}$ with similar efficiency whereas HPV16 E7 binds and causes degradation of $617 \mathrm{RB} 1$ but not $\mathrm{RB} 1^{\mathrm{L}}$. The results shown are representative of two independent experiments. A 618 cartoon of the $\mathrm{RB} 1^{\mathrm{L}}$ mutant is shown at the top of the figure. 
623

624

625

626

627

628

629

630

631

632

633

634

635

636

637

638

639

640

641

642

643

644

645

646

647

mJ UVB the next day and then monitored for papilloma formation over 4 months. MmuPV1 induced warts in wild type and $\mathrm{RB} 1^{\mathrm{L}}$ mutant $\mathrm{FVB} / \mathrm{N}$ mice with a similar incidence (Fisher's exact test, $\mathrm{p}$ Value $=1$, two-sided) (A). Warts arising in wild-type and $\mathrm{RB} 1^{\mathrm{L}}$ mutant $\mathrm{FVB} / \mathrm{N}$ mice share similar microscopic features. Warts from both mouse genotypes were harvested, serially sectioned, and stained with hematoxylin and eosin (H\&E), processed to detect Ki67 (Ki67, red; DAPI, blue) by immunofluorescence, and MCM7 by immunohistochemistry (B).

\section{Figure 6. The C-terminal domain of RB1 is necessary for MmuPV1 E7 binding.}

Schematic representation of the RB1 protein and the expression plasmids used (A). HA-epitope tagged versions of full-length RB1, and the two truncation mutants 1-792 and 379-928 were expressed in SAOS-2 human osteosarcoma cells in combination with FLAG/HA epitope-tagged MmuPV1 (M E7) and HPV16 E7 (16 E7) expression vectors. After immunoprecipitation with FLAG antibodies, HA-tagged E7 and co-precipitated RB1 proteins were detected by HA immunoblot. The result shown is representative of two independent experiments. (B).

\section{Figure 7. The RB1 binding site maps the MmuPV1 E7 C-terminus. Sequence alignment} of the C-terminal domains of MmuPV1, canine papillomavirus 2 (CPV2), $\gamma 1$-HPV4, $\gamma 24-H P V 197$, $\beta 1$-HPV8, and $\alpha 9-H P V 16$ E7. Identical residues are marked by black boxes, chemically similar residues are shaded in gray. The position of the CXXC motifs that form a zinc-binding site is shown. The position of the aspartate residue at position 90 (D90) that is important for RB1 binding is indicated by a red box (A). Various FLAG/HA-tagged MmuPV1 E7 mutants were expressed in HCT116 cells and co-precipitated RB1 was detected by immunoblotting. The result shown is representative of six independent experiments (B). Immunoprecipitation western blot analyses to assess $\mathrm{RB} 1$ binding by various MmuPV1 $E 7^{\mathrm{D} 90}$ mutants. The result shown is representative of two independent experiments (C). Immunoprecipitation/western blot analysis 
648

649

650

651

652

653

654

655

656

657

658

659

660

661

662

663

664

665

666

667

668

669

670

671

672

673

documenting that MmuPV1 E7 $7^{\mathrm{D} 90 \mathrm{~A}}$ is defective for binding to murine Rb1. Wild type MmuPV1 E7 and the D90A mutant were transiently expressed in NIH3T3 cells and binding assessed by immunoprecipitation/western blotting. The blot shown is representative of 2 independent experiments (D). Quantifications of E7-coprecipitated RB1 or Rb1 are normalized to the amount of E7 that is precioitated and are shown underneath.

\section{Figure 8. MmuPV1 $\mathrm{E} 7^{\mathrm{Dg0A}}$ virus gives rise to reduced incidence and smaller-sized} warts than wild type MmuPV1. To assess infectivity of quasivirus stocks, mouse JB6 keratinocytes were exposed to quasivirus and 48 hours later RNA was extracted and subjected to reverse transcription-coupled polymerase chain reaction (RT-PCR) to detect MmuPV1 E1^E4 (top panel) or p53 (bottom panel) transcripts. Shown are results for mock-infected cells, and cells infected with equal amounts of MmuPV1 derived from warts (positive control), wild type MmuPV1 quasivirus (wild type), and two stocks of $E 7^{\mathrm{D} 90 \mathrm{~A}}$ mutant quasivirus $\left(\mathrm{E} 7^{\mathrm{D} 90 \mathrm{~A}}\right)(\mathrm{A})$. Wart incidence arising at sites on nude mice infected with $10^{8} \mathrm{VGE}$ of wild type MmuPV1 quasivirus, 107 VGE of wild type MmuPV1 quasivirus as well as $10^{8}$ or $10^{7}$ VGE, respectively, of two independent preparations of MmuPV1 $E 7^{\mathrm{D} 90 \mathrm{~A}}$ quasivirus (E7 $7^{\mathrm{D} 90 \mathrm{~A}}$ Stock 1, $E 7^{\mathrm{D} 90 \mathrm{~A}}$ Stock 2). The incidence of warts at sites infected with MmuPV1 E7 ${ }^{\mathrm{D} 90 \mathrm{~A}}$ quasivirus was significantly less compared to dose equivalent of wild type MmuPV1 quasivirus (Fisher's exact test, two-sided: MmuPV1 (10 ${ }^{8}$ VGE) vs. E7 ${ }^{\text {D90A }}$ Stock 1, p<0.0001; MmuPV1 $\left(10^{7}\right.$ VGE) vs. E7 ${ }^{\text {D90A }}$ Stock 2 , $p<0.001)$. A dosage effect in wart formation was also observed with the wildtype MmuPV1 (MmuPV1 (10 ${ }^{8}$ VGE) vs. MmuPV1 (10 VGE), $\left.p=0.0001\right)$ (B). Kaplan-Meier plot showing that the percent of wart free sites over time is significantly different for MmuPV1 versus MmuPV1 E7 ${ }^{\mathrm{D} 90 \mathrm{~A}}$ quasivirus infections (LogRank test (two-sided): MmuPV1 (10 ${ }^{8}$ VGE) vs. MmuPV1 E7 ${ }^{\mathrm{D} 90 \mathrm{~A}}$ Stock 1, $p<0.0001$; MmuPV1 (107 VGE) versus MmuPV1 E7 ${ }^{\text {D90A }}$ Stock 2, $p<0.0001$; MmuPV1 $\left(10^{8} \mathrm{VGE}\right)$ versus MmuPV1 $\left.\left(10^{7} \mathrm{VGE}\right), \mathrm{p}<0.001\right)$ (C). Representative images and of tails of mice infected with the different quasiviruses at the 4 months endpoint (top images), along with equal 
674 magnification of scanned images of sections of tails harboring representative warts stained with

675 H\&E (bottom images) (D). Size of warts at the 4 months endpoint. Warts arising from the

676 MmuPV1 E7 ${ }^{\text {D90A }}$ quasivirus were significantly smaller (T-test, two-sided: MmuPV1 versus

677 MmuPV1 E7 $\left.7^{\mathrm{D} 90 \mathrm{~A}}, \mathrm{p}=0.003\right)$. Six MmuPV1 warts (three from each dose) and all three MmuPV1

$678 E 7^{\mathrm{D} 90 \mathrm{~A}}$ quasivirus induced warts (two from Stock 1 and one from Stock 2) were used for this

679 quantification.

680

681 Figure 9. MmuPV1 E7 $7^{\mathrm{D} 90 \mathrm{~A}}$ quasivirus-induced warts display similar histological

682 features as warts induced by wildtype MmuPV1. Shown are serial sections of the mock-

683 infected tail (left column), and tail warts induced by wildtype MmuPV1 (middle column) or

684 MmuPV1 E7 ${ }^{\mathrm{D} 90 \mathrm{~A}}$ quasivirus (right column) stained with H\&E (A), stained for MmuPV1 L1 (red),

$685 \mathrm{~K} 14$ (green) by immunofluorescence and counterstained with DAPI (blue) (B), or

686 immunohistochemically stained for BrdU (C), or MCM7 (D).

687 


\section{Table Legends:}

689

690 Table S1: Cellular proteins identified by Affinity purification/mass spectrometry (AP/MS) analyses of

691 carboxyl- or amino terminally epitope tagged MmuPV1 E7 (CE7 and NE7 respectively). The number of

692 unique and total peptides identified for each protein are shown. See reference [17] for experimental

693 details.

694

695 Table S2: Table S2: Sequences of PCR primers used in this study. 


\section{REFERENCES}

1. Van Doorslaer K, Li Z, Xirasagar S, Maes P, Kaminsky D, Liou D, Sun Q, Kaur R, Huyen Y, McBride AA. 2017. The Papillomavirus Episteme: a major update to the papillomavirus sequence database (pave.niaid.nih.gov). Nucleic Acids Res 45:D499-D506.

2. Schiffman M, Castle PE, Jeronimo J, Rodriguez AC, Wacholder S. 2007. Human papillomavirus and cervical cancer. Lancet 370:890-907.

3. Chaturvedi AK, Engels EA, Pfeiffer RM, Hernandez BY, Xiao W, Kim E, Jiang B, Goodman MT, Sibug-Saber M, Cozen W, Liu L, Lynch CF, Wentzensen N, Jordan RC, Altekruse S, Anderson WF, Rosenberg PS, Gillison ML. 2011. Human papillomavirus and rising oropharyngeal cancer incidence in the United States. J Clin Oncol 29:4294-301.

4. de Martel C, Ferlay J, Franceschi S, Vignat J, Bray F, Forman D, Plummer M. 2012. Global burden of cancers attributable to infections in 2008: a review and synthetic analysis. Lancet Oncol 13:607-15.

5. Fuchs PG, Iftner T, Weninger J, Pfister H. 1986. Epidermodysplasia verruciformis-associated human papillomavirus 8: genomic sequence and comparative analysis. J Virol 58:626-34.

6. Zachow KR, Ostrow RS, Faras AJ. 1987. Nucleotide sequence and genome organization of human papillomavirus type 5. Virology 158:251-4.

7. Howley PM, Pfister HJ. 2015. Beta genus papillomaviruses and skin cancer. Virology 479480:290-6.

8. Meyers JM, Munger K. 2014. The viral etiology of skin cancer. J Invest Dermatol 134:E29-32.

9. Tommasino M. 2017. The biology of beta human papillomaviruses. Virus Res 231:128-138.

10. Vande Pol SB, Klingelhutz AJ. 2013. Papillomavirus E6 oncoproteins. Virology 445:115-37.

11. Roman A, Munger K. 2013. The papillomavirus E7 proteins. Virology 445:138-68.

12. Moody CA, Laimins LA. 2010. Human papillomavirus oncoproteins: pathways to transformation. Nat Rev Cancer 10:550-60.

13. Meyers JM, Grace M, Uberoi A, Lambert PF, Munger K. 2018. Inhibition of TGF-beta and NOTCH Signaling by Cutaneous Papillomaviruses. Front Microbiol 9:389.

14. Spurgeon ME, Lambert PF. 2020. Mus musculus Papillomavirus 1: a New Frontier in Animal Models of Papillomavirus Pathogenesis. J Virol 94.

15. Meyers JM, Uberoi A, Grace M, Lambert PF, Munger K. 2017. Cutaneous HPV8 and MmuPV1 E6 Proteins Target the NOTCH and TGF-beta Tumor Suppressors to Inhibit Differentiation and Sustain Keratinocyte Proliferation. PLoS Pathog 13:e1006171.

16. Wang J, Zhou D, Prabhu A, Schlegel R, Yuan H. 2010. The canine papillomavirus and gamma HPV E7 proteins use an alternative domain to bind and destabilize the retinoblastoma protein. PLoS Pathog 6:e1001089.

17. Grace M, Munger K. 2017. Proteomic analysis of the gamma human papillomavirus type 197 E6 and $\mathrm{E7}$ associated cellular proteins. Virology 500:71-81.

18. Xue XY, Majerciak V, Uberoi A, Kim BH, Gotte D, Chen X, Cam M, Lambert PF, Zheng ZM. 2017. The full transcription map of mouse papillomavirus type 1 (MmuPV1) in mouse wart tissues. PLoS Pathog 13:e1006715.

19. Pyeon D, Lambert PF, Ahlquist P. 2005. Production of infectious human papillomavirus independently of viral replication and epithelial cell differentiation. Proc Natl Acad Sci U S A 102:9311-6.

20. Uberoi A, Yoshida S, Frazer IH, Pitot HC, Lambert PF. 2016. Role of Ultraviolet Radiation in Papillomavirus-Induced Disease. PLoS Pathog 12:e1005664. 
21. Cladel NM, Budgeon LR, Cooper TK, Balogh KK, Hu J, Christensen ND. 2013. Secondary infections, expanded tissue tropism, and evidence for malignant potential in immunocompromised mice infected with Mus musculus papillomavirus 1 DNA and virus. J Virol 87:9391-5.

22. Gonzalez SL, Stremlau M, He X, Basile JR, Munger K. 2001. Degradation of the retinoblastoma tumor suppressor by the human papillomavirus type 16 E7 oncoprotein is important for functional inactivation and is separable from proteasomal degradation of E7. J Virol 75:7583-91.

23. Shew JY, Lin BT, Chen PL, Tseng BY, Yang-Feng TL, Lee WH. 1990. C-terminal truncation of the retinoblastoma gene product leads to functional inactivation. Proc Natl Acad Sci U S A 87:6-10.

24. Boyer SN, Wazer DE, Band V. 1996. E7 protein of human papilloma virus-16 induces degradation of retinoblastoma protein through the ubiquitin-proteasome pathway. Cancer Res 56:4620-4.

25. Dyson N. 1998. The regulation of E2F by pRB-family proteins. Genes Dev 12:2245-62.

26. Nevins JR. 2001. The Rb/E2F pathway and cancer. Hum Mol Genet 10:699-703.

27. Weinberg RA. 1995. The retinoblastoma protein and cell cycle control. Cell 81:323-30.

28. Narasimha AM, Kaulich M, Shapiro GS, Choi YJ, Sicinski P, Dowdy SF. 2014. Cyclin D activates the $\mathrm{Rb}$ tumor suppressor by mono-phosphorylation. Elife 3.

29. Sanidas I, Morris R, Fella KA, Rumde PH, Boukhali M, Tai EC, Ting DT, Lawrence MS, Haas W, Dyson NJ. 2019. A Code of Mono-phosphorylation Modulates the Function of RB. Mol Cell 73:985-1000 e6.

30. Chellappan S, Kraus VB, Kroger B, Munger K, Howley PM, Phelps WC, Nevins JR. 1992. Adenovirus $\mathrm{E} 1 \mathrm{~A}$, simian virus 40 tumor antigen, and human papillomavirus $\mathrm{E} 7$ protein share the capacity to disrupt the interaction between transcription factor E2F and the retinoblastoma gene product. Proc Natl Acad Sci U S A 89:4549-53.

31. Lees E, Faha B, Dulic V, Reed SI, Harlow E. 1992. Cyclin E/cdk2 and cyclin A/cdk2 kinases associate with p107 and E2F in a temporally distinct manner. Genes Dev 6:1874-85.

32. Leone G, DeGregori J, Yan Z, Jakoi L, Ishida S, Williams RS, Nevins JR. 1998. E2F3 activity is regulated during the cell cycle and is required for the induction of $S$ phase. Genes Dev 12:212030.

33. Lee HH, Chiang WH, Chiang SH, Liu YC, Hwang J, Ng SY. 1995. Regulation of cyclin D1, DNA topoisomerase I, and proliferating cell nuclear antigen promoters during the cell cycle. Gene Expr 4:95-109.

34. Lee JO, Russo AA, Pavletich NP. 1998. Structure of the retinoblastoma tumour-suppressor pocket domain bound to a peptide from HPV E7. Nature 391:859-65.

35. Dick FA, Sailhamer E, Dyson NJ. 2000. Mutagenesis of the pRB pocket reveals that cell cycle arrest functions are separable from binding to viral oncoproteins. Mol Cell Biol 20:3715-27.

36. Balsitis S, Dick F, Dyson N, Lambert PF. 2006. Critical roles for non-pRb targets of human papillomavirus type 16 E7 in cervical carcinogenesis. Cancer Res 66:9393-400.

37. Balsitis S, Dick F, Lee D, Farrell L, Hyde RK, Griep AE, Dyson N, Lambert PF. 2005. Examination of the $p R b$-dependent and $p R b$-independent functions of $E 7$ in vivo. J Virol 79:11392-402.

38. Brake T, Connor JP, Petereit DG, Lambert PF. 2003. Comparative analysis of cervical cancer in women and in a human papillomavirus-transgenic mouse model: identification of minichromosome maintenance protein 7 as an informative biomarker for human cervical cancer. Cancer Res 63:8173-80.

39. Spurgeon ME, Uberoi A, McGregor SM, Wei T, Ward-Shaw E, Lambert PF. 2019. A Novel In Vivo Infection Model To Study Papillomavirus-Mediated Disease of the Female Reproductive Tract. mBio 10.

40. Joh J, Jenson AB, Proctor M, Ingle A, Silva KA, Potter CS, Sundberg JP, Ghim SJ. 2012. Molecular diagnosis of a laboratory mouse papillomavirus (MusPV). Exp Mol Pathol 93:416-21.

41. Uberoi A, Lambert PF. 2017. Rodent Papillomaviruses. Viruses 9. 
42. Patrick DR, Oliff A, Heimbrook DC. 1994. Identification of a novel retinoblastoma gene product binding site on human papillomavirus type 16 E7 protein. J Biol Chem 269:6842-50.

43. Todorovic B, Hung K, Massimi P, Avvakumov N, Dick FA, Shaw GS, Banks L, Mymryk JS. 2012. Conserved region 3 of human papillomavirus $16 \mathrm{E} 7$ contributes to deregulation of the retinoblastoma tumor suppressor. J Virol 86:13313-23.

44. Brandsma JL, Yang ZH, Barthold SW, Johnson EA. 1991. Use of a rapid, efficient inoculation method to induce papillomas by cottontail rabbit papillomavirus DNA shows that the E7 gene is required. Proc Natl Acad Sci U S A 88:4816-20.

45. Meyers C, Harry J, Lin YL, Wettstein FO. 1992. Identification of three transforming proteins encoded by cottontail rabbit papillomavirus. J Virol 66:1655-64.

46. Haskell KM, Vuocolo GA, Defeo-Jones D, Jones RE, Ivey-Hoyle M. 1993. Comparison of the binding of the human papillomavirus type 16 and cottontail rabbit papillomavirus $E 7$ proteins to the retinoblastoma gene product. J Gen Virol 74 ( Pt 1):115-9.

47. Defeo-Jones D, Vuocolo GA, Haskell KM, Hanobik MG, Kiefer DM, McAvoy EM, Ivey-Hoyle M, Brandsma JL, Oliff A, Jones RE. 1993. Papillomavirus E7 protein binding to the retinoblastoma protein is not required for viral induction of warts. J Virol 67:716-25.

48. Qin XQ, Chittenden T, Livingston DM, Kaelin WG, Jr. 1992. Identification of a growth suppression domain within the retinoblastoma gene product. Genes Dev 6:953-64.

49. Sellers WR, Rodgers JW, Kaelin WG, Jr. 1995. A potent transrepression domain in the retinoblastoma protein induces a cell cycle arrest when bound to E2F sites. Proc Natl Acad Sci U S A 92:11544-8.

50. Welch PJ, Wang JY. 1995. Disruption of retinoblastoma protein function by coexpression of its C pocket fragment. Genes Dev 9:31-46.

51. Welch PJ, Wang JY. 1993. A C-terminal protein-binding domain in the retinoblastoma protein regulates nuclear c-Abl tyrosine kinase in the cell cycle. Cell 75:779-90.

52. Ji P, Jiang H, Rekhtman K, Bloom J, Ichetovkin M, Pagano M, Zhu L. 2004. An Rb-Skp2-p27 pathway mediates acute cell cycle inhibition by $\mathrm{Rb}$ and is retained in a partial-penetrance $\mathrm{Rb}$ mutant. Mol Cell 16:47-58.

53. Julian LM, Palander O, Seifried LA, Foster JE, Dick FA. 2008. Characterization of an E2F1-specific binding domain in $P R B$ and its implications for apoptotic regulation. Oncogene 27:1572-9.

54. Ishak CA, Marshall AE, Passos DT, White CR, Kim SJ, Cecchini MJ, Ferwati S, MacDonald WA, Howlett CJ, Welch ID, Rubin SM, Mann MRW, Dick FA. 2016. An RB-EZH2 Complex Mediates Silencing of Repetitive DNA Sequences. Mol Cell 64:1074-1087.

55. Binne UK, Classon MK, Dick FA, Wei W, Rape M, Kaelin WG, Jr., Naar AM, Dyson NJ. 2007. Retinoblastoma protein and anaphase-promoting complex physically interact and functionally cooperate during cell-cycle exit. Nat Cell Biol 9:225-32.

56. Dick FA, Goodrich DW, Sage J, Dyson NJ. 2018. Non-canonical functions of the RB protein in cancer. Nat Rev Cancer 18:442-451.

57. Xiao B, Spencer J, Clements A, Ali-Khan N, Mittnacht S, Broceno C, Burghammer M, Perrakis A, Marmorstein R, Gamblin SJ. 2003. Crystal structure of the retinoblastoma tumor suppressor protein bound to E2F and the molecular basis of its regulation. Proc Natl Acad Sci U S A 100:2363-8.

58. Lee C, Chang JH, Lee HS, Cho Y. 2002. Structural basis for the recognition of the E2F transactivation domain by the retinoblastoma tumor suppressor. Genes Dev 16:3199-212.

59. Joh J, Jenson AB, King W, Proctor M, Ingle A, Sundberg JP, Ghim SJ. 2011. Genomic analysis of the first laboratory-mouse papillomavirus. J Gen Virol 92:692-8. 
60. Kiyono T, Foster SA, Koop JI, McDougall JK, Galloway DA, Klingelhutz AJ. 1998. Both $\mathrm{Rb} / \mathrm{p} 16 \mathrm{INK} 4 \mathrm{a}$ inactivation and telomerase activity are required to immortalize human epithelial cells. Nature 396:84-8.

61. Jeon S, Allen-Hoffmann BL, Lambert PF. 1995. Integration of human papillomavirus type 16 into the human genome correlates with a selective growth advantage of cells. J Virol 69:2989-97.

62. Jeon S, Lambert PF. 1995. Integration of human papillomavirus type 16 DNA into the human genome leads to increased stability of E6 and E7 mRNAs: implications for cervical carcinogenesis. Proc Natl Acad Sci U S A 92:1654-8.

63. Chapman S, Liu X, Meyers C, Schlegel R, McBride AA. 2010. Human keratinocytes are efficiently immortalized by a Rho kinase inhibitor. J Clin Invest 120:2619-26.

64. Spangle JM, Munger K. 2010. The human papillomavirus type 16 E6 oncoprotein activates mTORC1 signaling and increases protein synthesis. J Virol 84:9398-407.

65. Baker SJ, Markowitz S, Fearon ER, Willson JK, Vogelstein B. 1990. Suppression of human colorectal carcinoma cell growth by wild-type p53. Science 249:912-5.

66. Halbert CL, Demers GW, Galloway DA. 1991. The E7 gene of human papillomavirus type 16 is sufficient for immortalization of human epithelial cells. J Virol 65:473-8.

67. Muller H, Lukas J, Schneider A, Warthoe P, Bartek J, Eilers M, Strauss M. 1994. Cyclin D1 expression is regulated by the retinoblastoma protein. Proc Natl Acad Sci U S A 91:2945-9.

68. Morgenstern JP, Land H. 1990. A series of mammalian expression vectors and characterisation of their expression of a reporter gene in stably and transiently transfected cells. Nucleic Acids Res 18:1068.

69. Longo PA, Kavran JM, Kim MS, Leahy DJ. 2013. Transient mammalian cell transfection with polyethylenimine (PEI). Methods Enzymol 529:227-40.

70. Isaac CE, Francis SM, Martens AL, Julian LM, Seifried LA, Erdmann N, Binne UK, Harrington L, Sicinski $P$, Berube NG, Dyson NJ, Dick FA. 2006. The retinoblastoma protein regulates pericentric heterochromatin. Mol Cell Biol 26:3659-71.

71. Handisurya A, Day PM, Thompson CD, Buck CB, Kwak K, Roden RB, Lowy DR, Schiller JT. 2012. Murine skin and vaginal mucosa are similarly susceptible to infection by pseudovirions of different papillomavirus classifications and species. Virology 433:385-94.

72. Handisurya A, Day PM, Thompson CD, Buck CB, Pang YY, Lowy DR, Schiller JT. 2013. Characterization of Mus musculus papillomavirus 1 infection in situ reveals an unusual pattern of late gene expression and capsid protein localization. J Virol 87:13214-25.

73. Hopman AH, Ramaekers FC, Speel EJ. 1998. Rapid synthesis of biotin-, digoxigenin-, trinitrophenyl-, and fluorochrome-labeled tyramides and their application for In situ hybridization using CARD amplification. J Histochem Cytochem 46:771-7. 


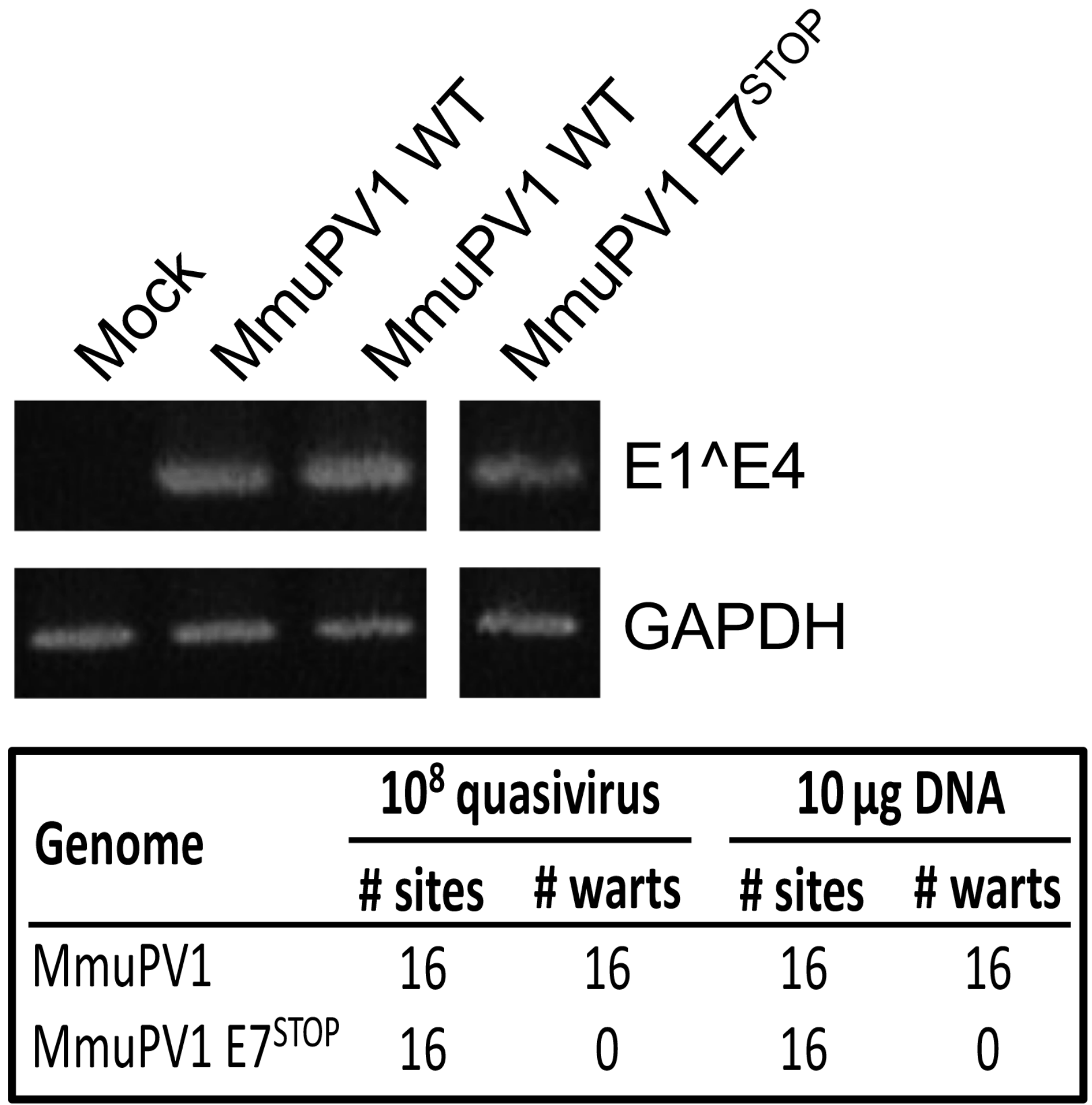




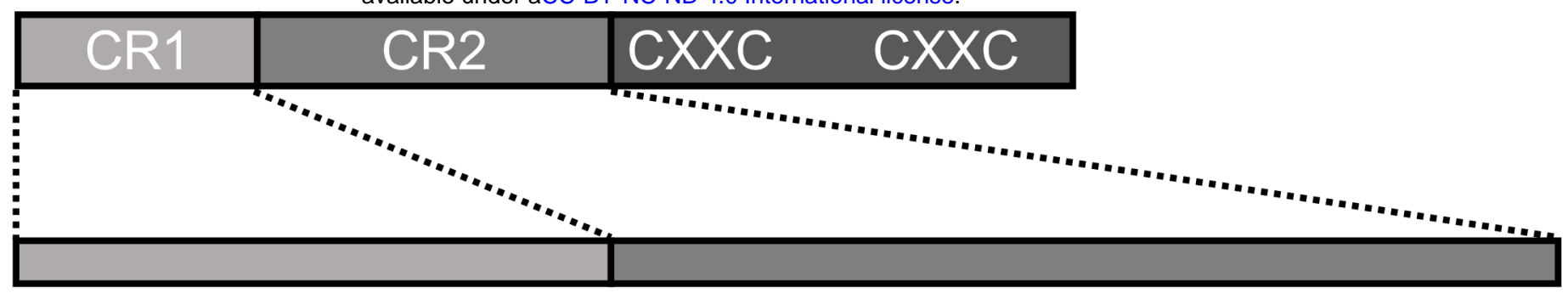

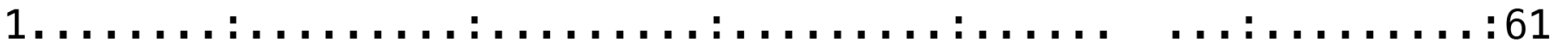

MmuPV1 E7

CPV2 E7

HPV4 E7

HPV197 E7

HPV8 E7

HPV16 E7

MQGPLPTIADIEIQNLDSLLGVGEPDLPDVGSSSLSPDSLGEEEEL-_ELETIDVDPYRIKTT
MRGSSPIIRDIDLELEELVLPAN--LLSGETL_--_-_ETEEEELOREPGRYRVVTL----MRGAAPTVADLNLELNDLVLPAN--LLSEEVLQSSDDEYEITEEESVVPFR-IDTC

B

Flag (E7)

Flag (E7)

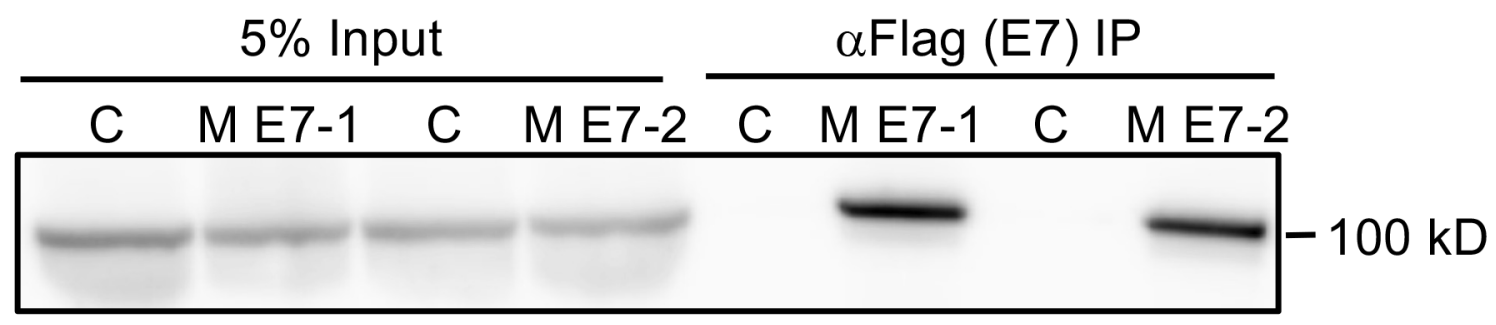

\begin{tabular}{|c|c|c|c|}
\hline $5 \%$ & nput & \multicolumn{2}{|c|}{$\alpha \mathrm{HA}(\mathrm{E} 7) \mathrm{IP}$} \\
\hline $\mathrm{C}$ & M E7 & C & M E7 \\
\hline $8=$ & $2=$ & & $=$ \\
\hline
\end{tabular}

MHGERATIRDVVLELT--_----KVACNSEVLFEESLSSDSEEEHEIFKIET--R-

M̄IGKEVTVQDFVLKLSEIQPEVLPVDLLCEEELPNEQETEEELD-----IE-

RTVFKIVAPCG -DLQPETT-DLYCYEQLNDSSEEEDEIDGPAGQAE-PDRAHYNIVTF-LXCXE

RB1

$\mathrm{Rb} 1$

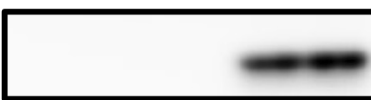

$-1$

$-20 \mathrm{kD}$

$\mathrm{D}$

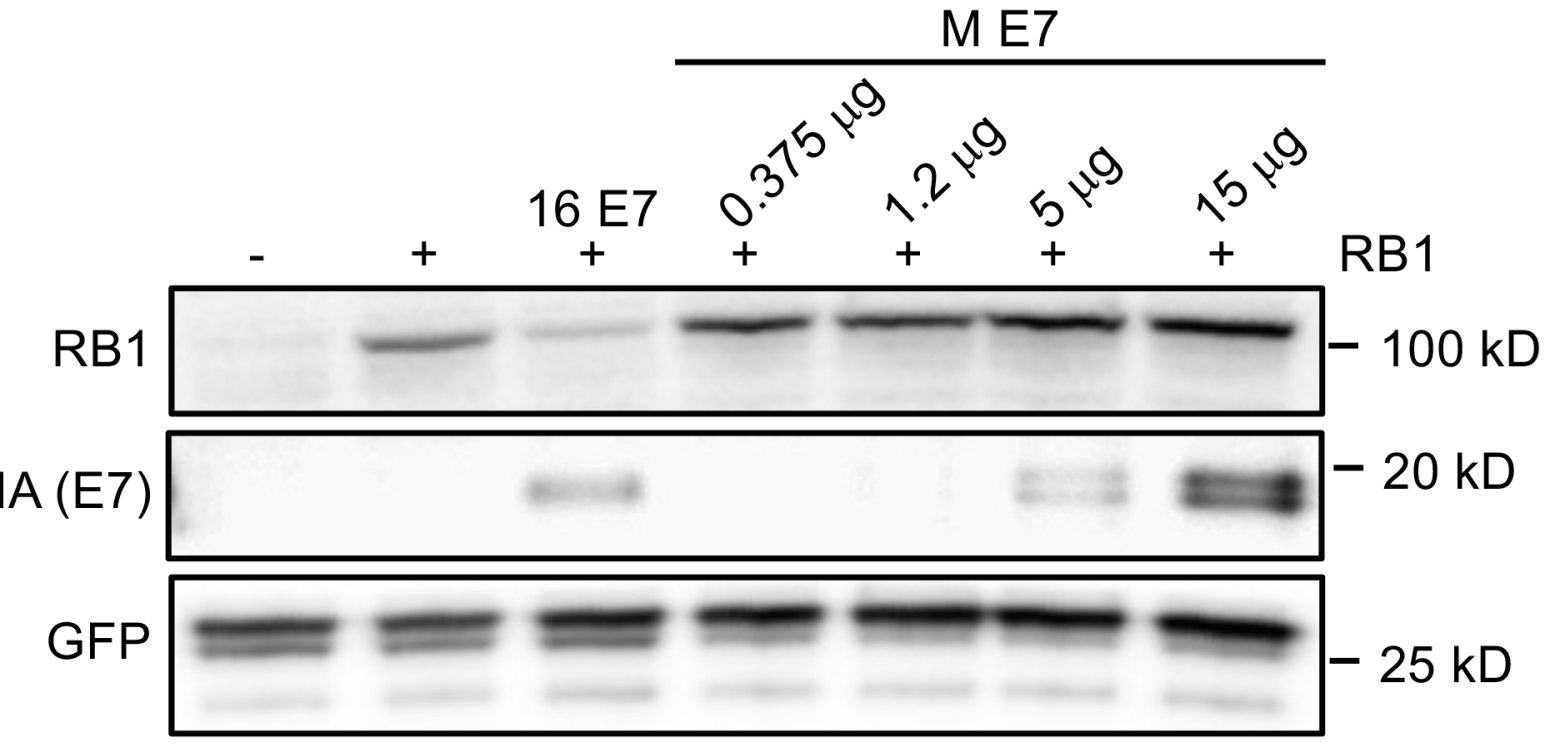



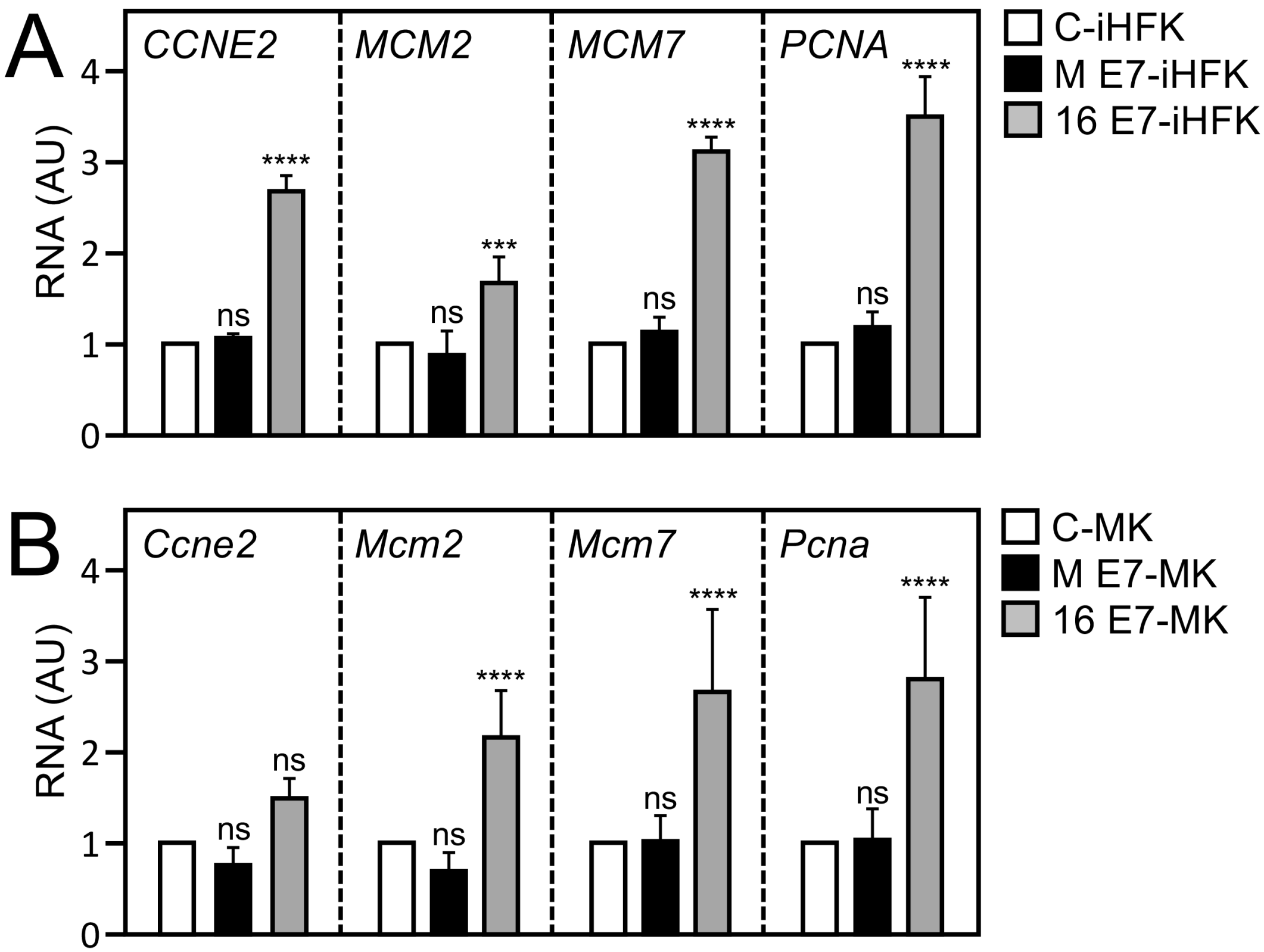
bioRxiv preprint doi: https://doi.org/10.1101/2021.07.30.454561; this version posted August 3, 2021. The copyright holder for this preprint (which was not certified by peer review) is the author/funder, who has granted bioRxiv a license to display the preprint in perpetuity. It is made available under aCC-BY-NC-ND 4.0 International license.

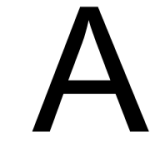

\begin{tabular}{|l|ccc|}
\hline & \# infected sites & \# warts & incidence \\
\hline FVB & 26 & 16 & $62 \%$ \\
RB1 ${ }^{\text {L }}$ mutant & 22 & 13 & $59 \%$ \\
\hline
\end{tabular}

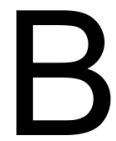

H\&E

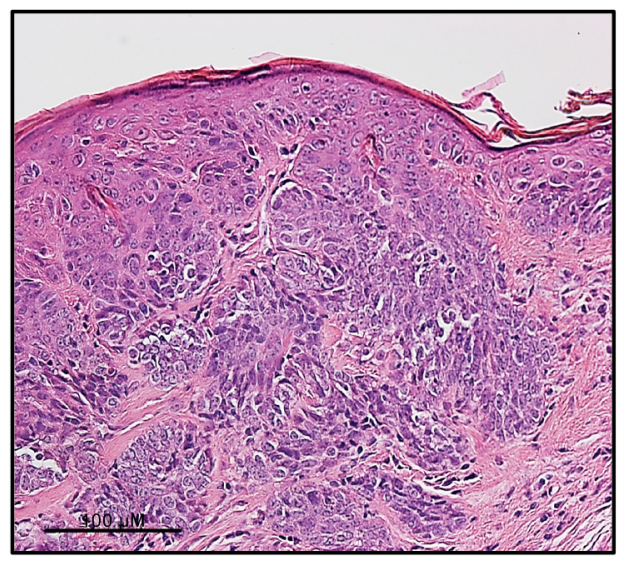

RB1L

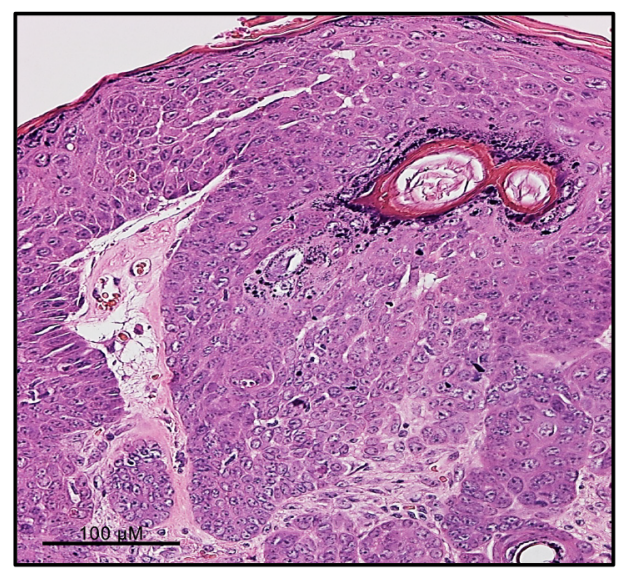

Ki67/DAPI
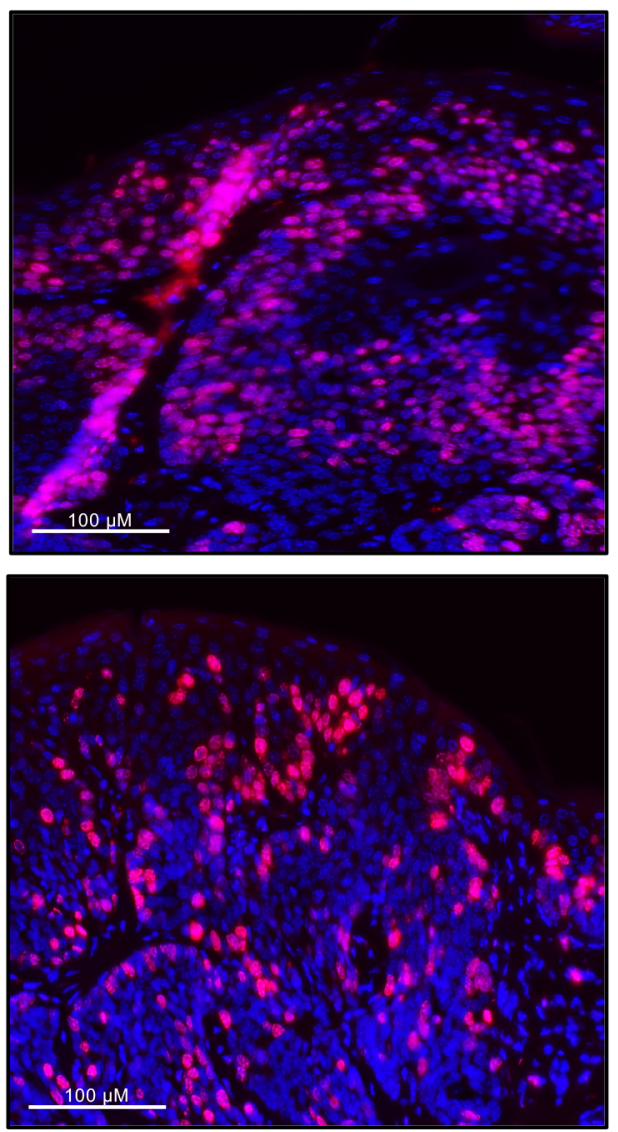

MCM7
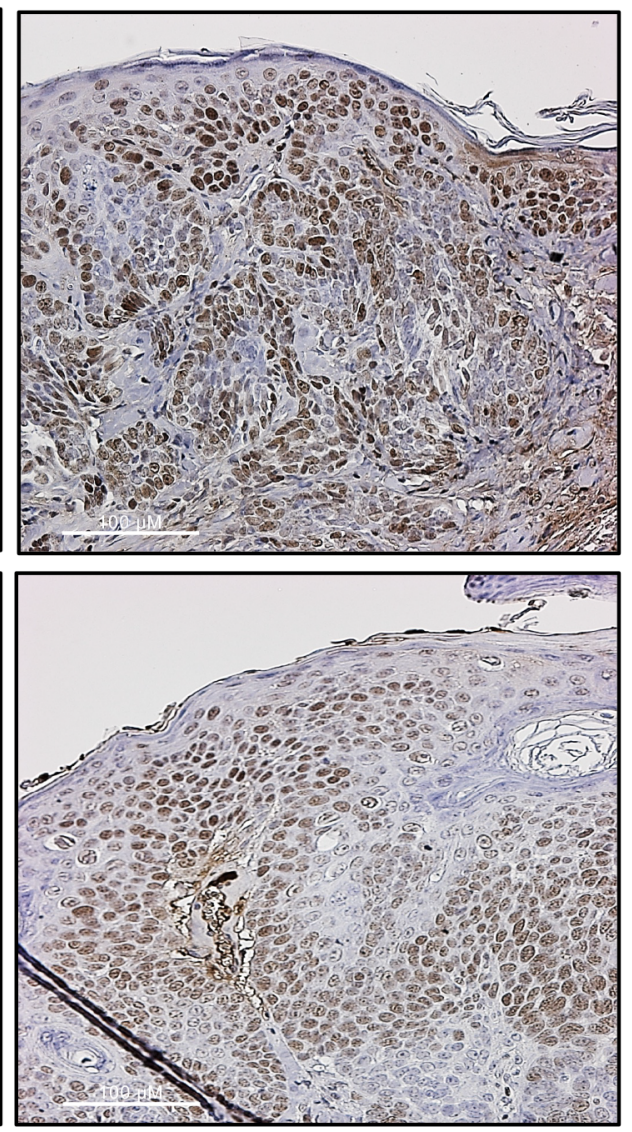

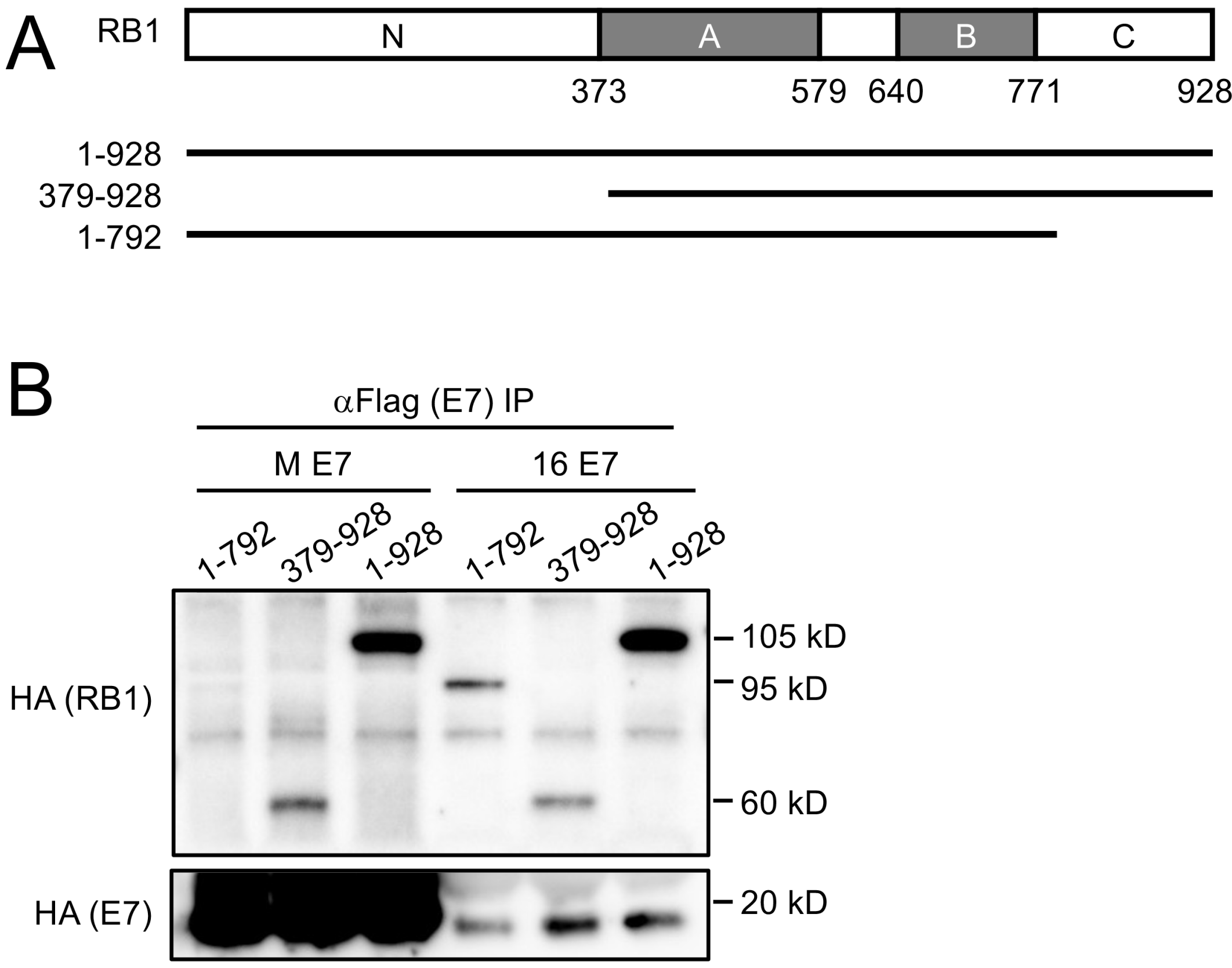


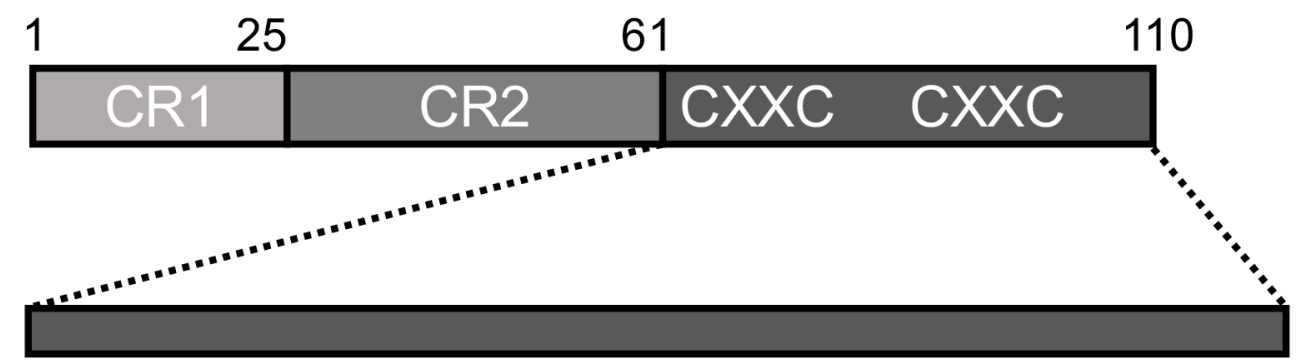

MmuPV1 E7

CPV2 E7

HPV4 E7

HPV197 E7

HPV8 E7

HPV16 E7

62

CFCCDTVLRFIIVTGDDSVKAFESLLLQD-LSFVCPHCVASYVNLRNGKR
CNICHSSLRLFVEVADESLIRLFQQLLLDGLGIICATCHKEHFSDGRRR
CYRCEVAVRITLYAAELGLRTLEQLLVEGKLTFCCTACARS--LNRNGR
CDNCRRGIRVCVRASNQGISRLQWLLEHD-IRFLCPACSRNLFQHGRFQ

CSCCQVKLRLFVNATDSGIRTFQELLFRD-LQLLCPECRGNCKHGGS

CCKCDSTLRLCVQSTHVDIRTLEDLLMGT-LGIVCPICSQKP

CXXC

CXXC
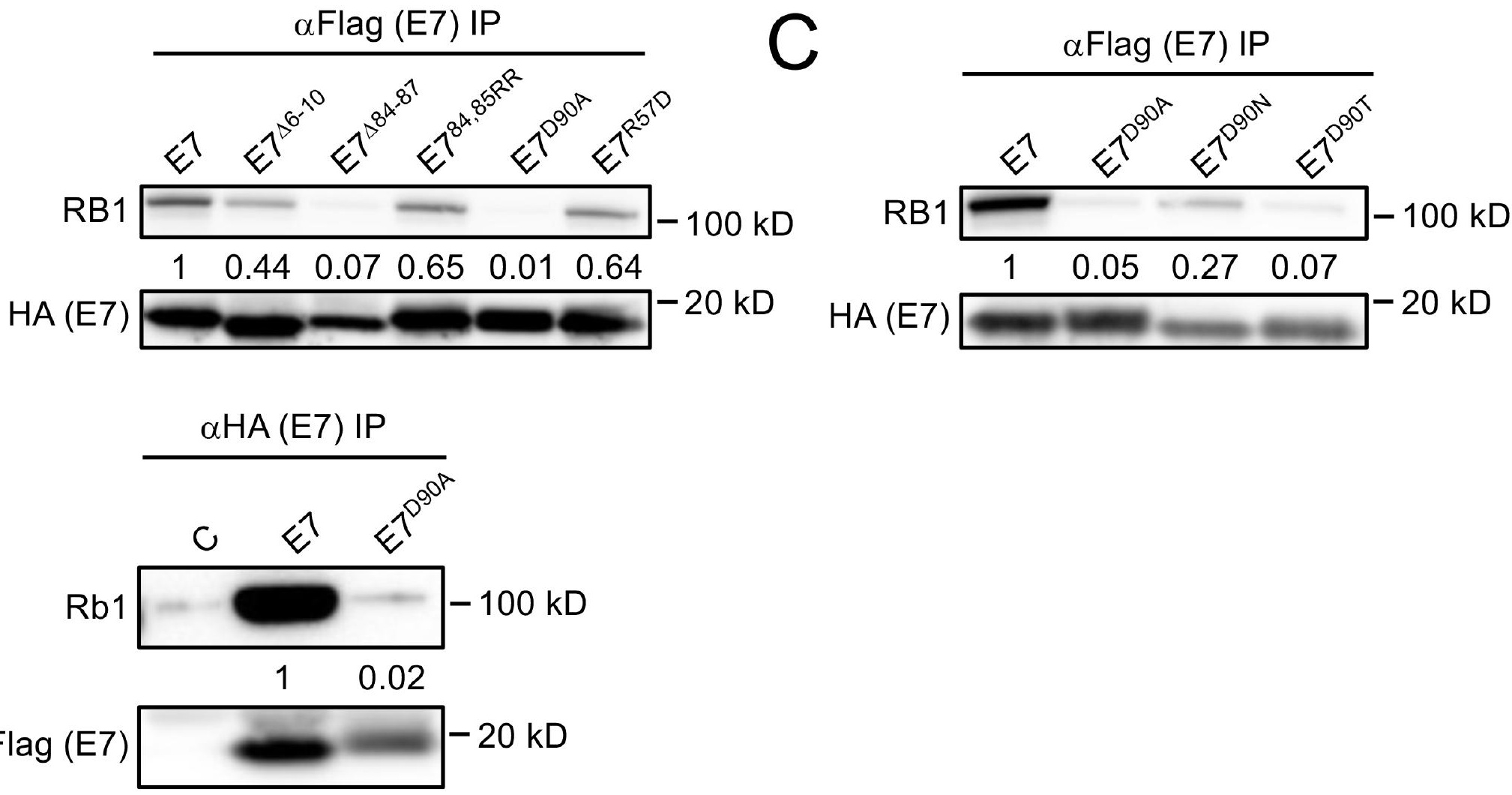
